\title{
Minimal PD-1 expression in mouse and human NK cells under diverse conditions
}

\author{
Sean J. Judge, ${ }^{1}$ Cordelia Dunai, ${ }^{2}$ Ethan G. Aguilar, ${ }^{2}$ Sarah C. Vick, ${ }^{3}$ Ian R. Sturgill, ${ }^{2}$ Lam T. Khuat, ${ }^{2}$ Kevin M. Stoffel, ${ }^{2}$ \\ Jonathan Van Dyke, ${ }^{4}$ Dan L. Longo, ${ }^{5}$ Morgan A. Darrow, ${ }^{6}$ Stephen K. Anderson, ${ }^{7}$ Bruce R. Blazar, ${ }^{8,9}$ Arta M. Monjazeb, ${ }^{10}$ \\ Jonathan S. Serody, ${ }^{11,12}$ Robert J. Canter, ${ }^{1}$ and William J. Murphy ${ }^{2,13}$
}

\begin{abstract}
'Department of Surgery and ${ }^{2}$ Department of Dermatology, UCD, Sacramento, California, USA. ${ }^{3}$ Department of Microbiology and Immunology, University of North Carolina at Chapel Hill, Chapel Hill, North Carolina, USA. ${ }^{4}$ Flow Cytometry Core, UCD, Sacramento, California, USA. ${ }^{5}$ Department of Medicine, Harvard Medical School, Boston, Massachusetts, USA. ${ }^{6}$ Department of Pathology and Laboratory Medicine, UCD, Sacramento, California, USA. ${ }^{7}$ Molecular Immunology Section, Leidos Biomedical Research Inc., Frederick National Laboratory for Cancer Research, Frederick, Maryland, USA. ${ }^{8}$ Masonic Cancer Center and ${ }^{9}$ Division of Blood and Bone Marrow Transplantation, Department of Pediatrics, University of Minnesota, Minneapolis, Minnesota, USA. ${ }^{10}$ Department of Radiation Oncology, UCD, Sacramento, California, USA. "'Lineberger Comprehensive Cancer Center and ${ }^{12}$ Department of Medicine, University of North Carolina at Chapel Hill, Chapel Hill, North Carolina, USA. ${ }^{13}$ Department of Medicine, UCD, Sacramento, California, USA.
\end{abstract}

\begin{abstract}
PD-1 expression is a hallmark of both early antigen-specific T cell activation and later chronic stimulation, suggesting key roles in both naive T cell priming and memory T cell responses. Although significant similarities exist between T cells and NK cells, there are critical differences in their biology and functions reflecting their respective adaptive and innate immune effector functions. Expression of PD-1 on NK cells is controversial despite rapid incorporation into clinical cancer trials. Our objective was to stringently and comprehensively assess expression of PD-1 on both mouse and human NK cells under multiple conditions and using a variety of readouts. We evaluated NK cells from primary human tumor samples, after ex vivo culturing, and from multiple mouse tumor and viral models using flow cytometry, quantitative reverse-transcriptase PCR (qRT-PCR), and RNA-Seq for PD-1 expression. We demonstrate that, under multiple conditions, human and mouse NK cells consistently lack PD-1 expression despite the marked upregulation of other activation/regulatory markers, such as TIGIT. This was in marked contrast to $\mathrm{T}$ cells, which were far more prominent within all tumors and expressed PD-1. These data have important implications when attempting to discern NK from T cell effects and to determine whether PD-1 targeting can be expected to have direct effects on NK cell functions.
\end{abstract}

\section{Introduction}

The introduction of immune checkpoint inhibition to cancer therapy targeting PD-1, PD-L1, and CTLA-4 has revolutionized clinical oncology with clear breakthrough advances in a growing list of hematologic and solid malignancies (1). These dramatic achievements have been built on key discoveries in the understanding and manipulation of $\mathrm{T}$ cell biology, particularly during chronic antigen exposure as well as in aging (2-4). As a result, the successes of immune checkpoint inhibition in reinvigorating exhausted $\mathrm{T}$ cells have generated vast efforts to increase the therapeutic reach

\section{Related Commentary: p. 2816}

Authorship note: RJC and WJM contributed equally to this work. Conflict of interest: BRB receives remuneration as an advisor to Kamon Pharmaceuticals, Five Prime Therapeutics, Regeneron Pharmaceuticals, Magenta Therapeutics, and BlueRock Therapeutics; receives research support from Fate Therapeutics, RXi Pharmaceuticals, Alpine Immune Sciences, Abbvie Inc., BlueRock Therapeutics Leukemia and Lymphoma Society, Children's Cancer Research Fund, and Kids First Fund; and is a cofounder of Tmunity.

Copyright: (5) 2020, American Society for Clinical Investigation.

Submitted: September 9, 2019; Accepted: February 26, 2020; Published: May 11, 2020. Reference information: / Clin Invest. 2020;130(6):3051-3068.

https://doi.org/10.1172/JCl133353. of targeting these pathways. As part of these efforts, investigators have examined the extent and role of PD-1 expression on other immune cells, including B cells $(5,6)$, innate lymphoid cells (7), myeloid-derived suppressor cells (8), and macrophages $(9,10)$. This search for PD-1 expression and associated therapeutic targeting has also been applied to NK cells (11), leading to speculation that PD-1 inhibition is a viable mechanism for augmenting the therapeutic potential of NK cells, particularly for tumors refractory to T cell-based approaches due to low MHCI expression (12).

NK cells have been characterized as key effectors in innate immunity and predominantly defined by their ability to kill virally infected and transformed host cells in an MHC-nonrestricted manner $(13,14)$. Due to their prevalence in hematopoietic tissues, studying their activity in hematologic malignancies (acute myeloid leukemia in particular) has been promising (15-19), but evidence for their efficacy and even roles against solid tumors is less robust. Recent attention has been directed toward the characterization of an NK "exhaustion" or "anergic" phenotype comparable to the well-characterized phenotype and functions attributed to T cells that appear to limit their efficacy (20-22). Recent studies have reported PD-1 expression, albeit variable and in some cases restricted to certain conditions, using both murine and human NK cells, and have suggested this molecule can have 
A

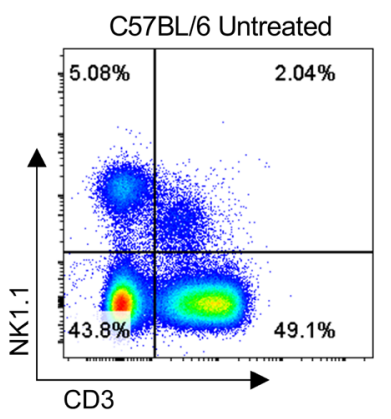

D

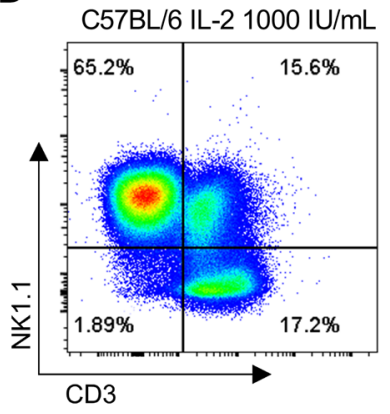

J $\quad$ Rag2/ $/ \mathrm{LL}-21000 \mathrm{IU} / \mathrm{mL}$

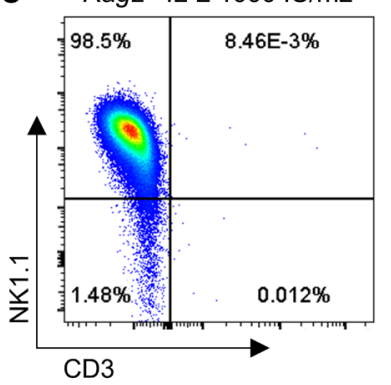

B

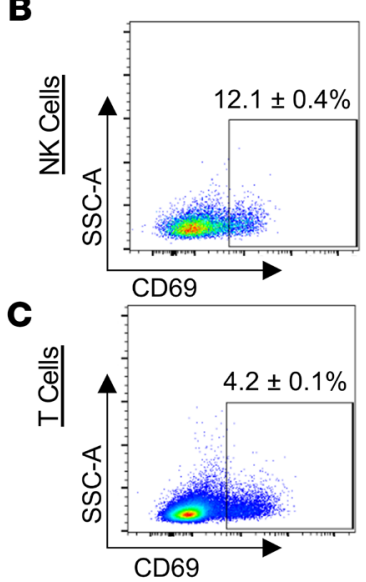

E

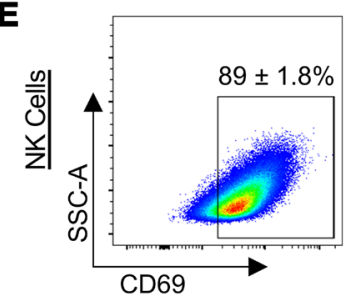

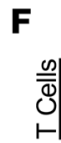

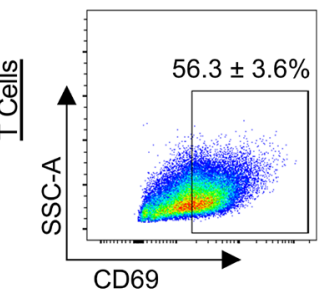

$\mathbf{K}$

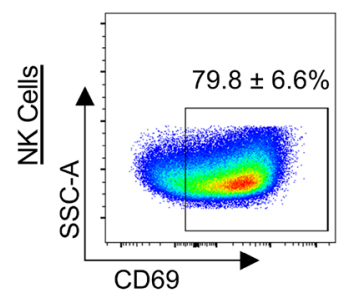

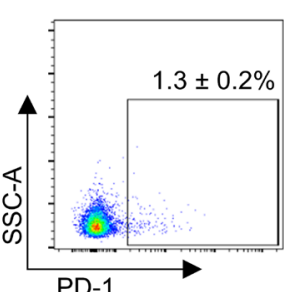
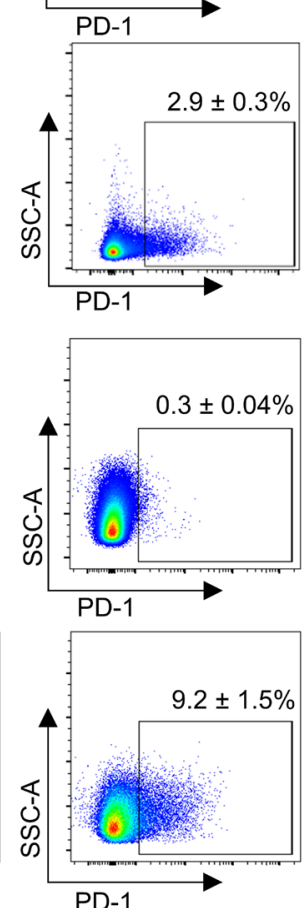

PD-1

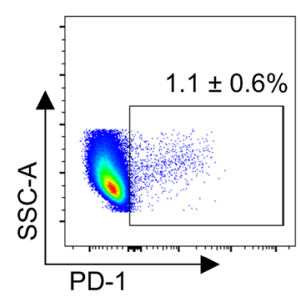

G

C57BL/6 ConA $2.5 \mu \mathrm{g} / \mathrm{mL}$

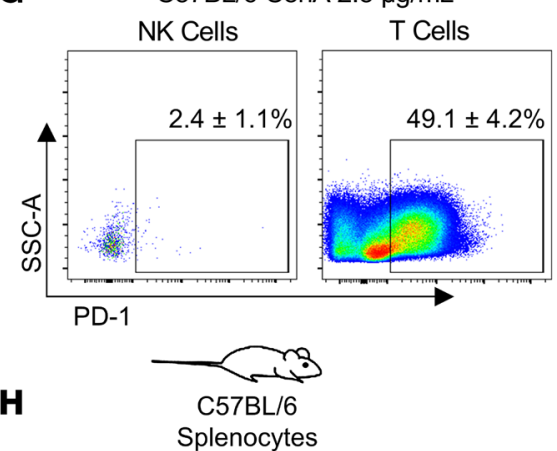

Splenocytes

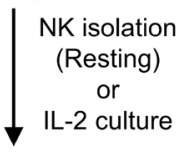

NK isolation

\&

RNA Sequencing

I

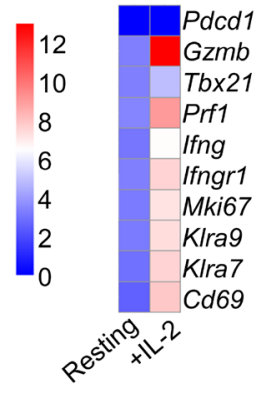

$\mathbf{L}$

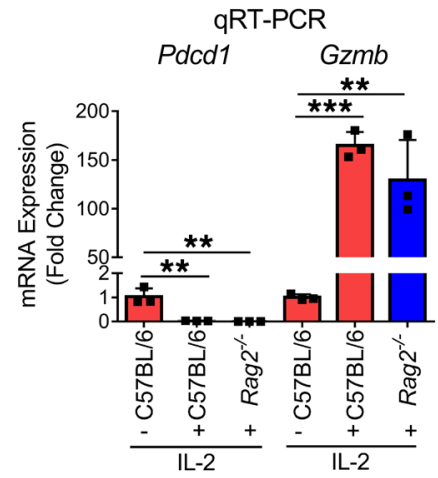

Figure 1. In vitro activated murine NK cells do not upregulate PD-1. (A) Representative NK1.1 and CD3 gating shows NK and T cell populations from resting C57BL/6 mice. (B and C) Untreated NK cells (CD3-NK1.1+) and T cells (CD3+NK1.1-) show low CD69 expression and minimal PD-1 expression. (D) ALAK cells were prepared by culturing splenocytes in $1000 \mathrm{IU} / \mathrm{mL}$ rhIL-2 for 7 days. Parent gating shows the enriched NK cell population. With cytokine activation, (E) NK cells show high CD69 expression, but still lack PD-1 expression, (F) while T cells exhibit moderate CD69 expression and low PD-1 expression. (G) Culture with T cell mitogen ConA leads to robust PD-1 expression on T cells, while PD-1 expression on NK cells remains minimal. (H) Schematic for RNA-Seq analysis of resting versus IL-2-stimulated sorted NK cells from WT splenocytes. (I) Despite marked upregulation of activation and proliferation markers, no expression of PD-1 is observed. (J) Rag2 $2^{-/-}$splenocytes were used to isolate pure NK cells. (K) NK cells are highly activated by CD69 expression, but PD-1 remains minimal. (L) Comparing WT ALAK cells and Rag2 $2^{-1-}$ ALAK cells to unstimulated WT splenocytes, mRNA expression of PD-1 was minimal on Rag2 $^{-/-}$NK cells, while expression of granzyme B (Gzmb) is upregulated. Data show mean \pm SD for $n=3-4 /$ group and are representative of 3 independent experiments. ${ }^{* *} P<0.01 ;{ }^{* *} P<0.001,1$-way ANOVA compared with unstimulated WT splenocytes.

a direct impact on NK cell responses $(12,23,24)$. To this end, antiPD-1 therapy is already being combined with various NK-promoting strategies to directly improve their clinical efficacy (25-28).

Despite these ongoing clinical efforts, the ability or extent of NK cells to express PD-1 remains controversial. Studies to date have reported seemingly contradictory results with varying out- comes for PD-1 expression on human NK cells and whether such PD-1 expression delineates NK cells with improved or diminished effector function along with similar reports regarding mouse NK cell expression (12, 23, 29-31). However, there are also opposing reports demonstrating lack of expression, but instead, prevalence of other immunoregulatory markers, such as TIGIT, PD-L1, TIM- 
3, and LAG-3 (31-34). As the majority of reports use solely flow cytometric analysis or one type of activation condition/model to ascertain expression, we sought to rigorously determine the extent of PD-1 expression on both human and mouse NK cells under highly relevant in vitro and in vivo conditions where NK cells are maximally activated. These included ex vivo cytokine stimulation of purified NK cell preparation, in vivo mouse cytomegalovirus (MCMV) infection, and assessment within the mouse and human intratumoral microenvironments. Using a combination of flow cytometric assessment, quantitative reverse-transcriptase PCR (qRT-PCR), and RNA-Seq of NK cells from WT, Rag2-- mice, and murine tumors as well as multiple patient-derived tumor specimens, we show that both human and murine NK cells express minimal PD-1 at baseline and that this does not increase in expression during diverse activation states. Our study demonstrates that PD-1 expression by NK cells is minimal and thus likely does not represent a direct pathway of NK immunoregulation.

\section{Results}

In vitro activated purified murine $N K$ cells do not express $P D-1$. We first sought to investigate the expression of PD-1 on highly activated and expanded mouse adherent lymphokine-activated killer (ALAK) cells, which are highly enriched for NK cells and cytotoxic $\mathrm{T}$ cells (35). Splenocytes were assessed with $\mathrm{NK}\left(\mathrm{NK}_{1.1}{ }^{+} \mathrm{CD}^{-}\right)$, $\mathrm{T}\left(\mathrm{NK} 1.1^{-} \mathrm{CD}^{+}\right)$, and $\mathrm{NKT}\left(\mathrm{NK} 1.1^{+} \mathrm{CD} 3^{+}\right)$cell populations shown in Figure $1 \mathrm{~A}$ and Supplemental Figure 1 (supplemental material available online with this article; https://doi.org/10.1172/ JCI133353DS1). Without stimulation, both NK and T cells exhibited low expression of the activation marker CD69 and less than 5\% PD-1 expression on the T cells, as young mice (2-4 months old), which have low numbers of memory $\mathrm{T}$ cells, were used (Figure 1, B and C). After culturing ALAKs for 7 days with $1000 \mathrm{IU} / \mathrm{mL}$ rhIL-2, there was a clear expansion of NK1.1 $1^{+} \mathrm{CD}^{-}{ }^{-} \mathrm{NK}$ cells (Figure 1D) with corresponding upregulation of CD69 observed on both NK and $\mathrm{T}$ cell populations. However, despite activation, there was no detection of PD- 1 on NK cells $(0.3 \% \pm 0.04 \%$ on NK cells compared with $9.2 \pm 1.5 \%$ on $\mathrm{T}$ cells) (Figure $1, \mathrm{E}$ and $\mathrm{F}$ ). In contrast, $\mathrm{T}$ cells stimulated with the mitogen concanavalin A (ConA) demonstrated a marked increase in PD-1 expression $(49.1 \% \pm 4.2 \%$ ) (Figure 1G). To confirm these flow cytometric findings, we sorted NK cells and assessed them by RNA-Seq analysis following activation with rhIL-2 (Figure 1H). These rhIL-2-activated NK cells showed marked upregulation of proliferation, activation, and functional NK-associated mRNA (granzyme B, perforin, IFN- $\gamma$, Ki67, CD69), but again no change in the minimal PD-1 mRNA expression detected (Figure 1I). Though PD-1 expression was negative by conventional flow cytometry and RNA-Seq, we also analyzed cultured splenocytes from B and T cell-deficient Rag2 ${ }^{-/-}$mice as a source of NK cells. As shown in Figure 1J, the NK cell population from cytokine-stimulated Rag2-- splenocytes was over 95\% NK1.1 $1^{+}$ cells compared with $0.01 \% \mathrm{CD}^{+}$cells. These ex vivo expanded NK cells demonstrated a high percentage of expression of CD69 (79.8\% $\pm 6.6 \%)$, but minimal PD-1 expression $(1.1 \% \pm 0.6 \%)$ (Figure $1 \mathrm{~K})$. Using qRT-PCR, we again detected minimal PD-1 mRNA expression in stimulated Rag2 ${ }^{--}$splenocytes compared with resting WT, whereas there was a greater than 100 -fold increase in mRNA expression of granzyme B in both WT and Rag2-- NK cell populations $(P<0.01$ for both, Figure $1 \mathrm{~L})$. Given recent reports of obesity promoting $\mathrm{T}$ cell exhaustion and PD-1 expression on T cells (36), we also analyzed spleens of diet-induced obese (DIO) mice and again observed no PD-1 expression on NK cells, despite significantly increased PD-1 expression on T cells (Supplemental Figure 2, A-C). Taken together, these data show that PD-1 is not appreciably expressed on mouse NK cells despite robust activation by rhIL-2 in vitro, as determined by flow cytometry, qRT-PCR, and RNA-Seq.

Mouse intratumoral NK cells do not appear in significant numbers in most mouse solid tumors and do not upregulate PD-1. Given the importance of murine tumor models in preclinical evaluation of cancer immunotherapy, we evaluated the extent of PD-1 expression on murine intratumoral NK cells. Overall, the reported expression of PD-1 on murine intratumoral NK cells has been variable, with some studies identifying PD-1 expression (albeit variable within groups of mice) on NK cells while others have not, potentially due to several experimental variables $(12,31)$. Given recent data suggesting that intratumoral NK cells in mice can express PD-1 and mediate antitumor effects when targeted (12), we first analyzed absolute numbers and relative percentages of $\mathrm{NK}$ and $\mathrm{T}$ cell infiltrates to assess overall immune cell prevalence in relation to tumor burden using a variety of mouse tumor lines. The gating strategy for each tumor model is shown to emphasize the heterogeneity between models and the relative contribution of $\mathrm{NK}$ and $\mathrm{T}$ cells to the immune and tumor infiltrates (Figure 2A and Supplemental Figure 3A). There was considerable heterogeneity within and between models regarding the total cells obtained (Figure 2B), reflective of tumor growth/progression differences, percentages of viable cells present (Figure 2C), and percentages of NK and $\mathrm{T}$ cells in the tumor (Figure 2D). However, in every tumor model examined, there was a clear paucity of intratumoral NK cells $\left(\mathrm{CD} 3{ }^{-} \mathrm{NKp} 46^{+}\right.$in BALB/c and CD3-NK1.1 ${ }^{+} \mathrm{NKp} 46^{+}$in C57BL/6 mice) relative to overall live tumor cells and to $\mathrm{T}$ cells (Figure 2D). For some mouse tumors, immune cell infiltration itself was minimal. Expression of CD 69 ( $58 \% \pm 2 \%$ vs. $32 \% \pm 4 \%$, $P=0.0002)$ and functional marker granzyme B $(58 \% \pm 14 \%$ vs. $12 \% \pm 5 \%, P=0.0001)$ on intratumoral NK cells compared with splenic NK cells suggested that the few NK cells present were in an activated state (Figure 2, E and F). However, regardless of tumor used, NK infiltration was low and PD-1 expression was minimal when compared with T cells in CT26 colon $(2 \% \pm 1 \%$ vs. $20 \% \pm$ $12 \%, P<0.0001), 4$ T1 breast ( $5 \% \pm 3 \%$ vs. $23 \% \pm 6 \%, P=0.0003)$, B16 melanoma ( $2 \% \pm 3 \%$ vs. $69 \% \pm 9 \%, P=0.0065)$, and 3LL lung tumors ( $7 \% \pm 5 \%$ vs. $37 \% \pm 8 \%, P<0.0001$ ) (Figure $2, \mathrm{G}$ and $\mathrm{H}$, and Supplemental Figure 3, B and C). Importantly, these results were observed regardless of the anti-PD-1 hybridoma clone used for analysis (Supplemental Figure 4, A and B). We then performed RNA-Seq analysis on sorted and isolated intratumoral NK and $\mathrm{T}$ cells. Tumors were dissociated (enzymatic digestion was not performed, as this can also affect CD3 expression, which is used as a critical marker to exclude $\mathrm{T}$ cells that readily express PD-1), then sorted for $\mathrm{CD}_{4} 5^{+} \mathrm{CD} 3^{-} \mathrm{NKp} 46^{+}$for $\mathrm{NK}$ cells and $\mathrm{CD} 45^{+}$ CD3 ${ }^{+} \mathrm{NKp} 46^{-}$for $\mathrm{T}$ cells (Figure 2I). Analysis revealed expected differences in gene expression for NK- and $\mathrm{T}$ cell-specific genes, including Gzma, Klrb1, Ncr1, Cd4, Cd8, and Foxp3, among others. Notably, there was again minimal expression of $P d c d 1$ (PD-1) 
A

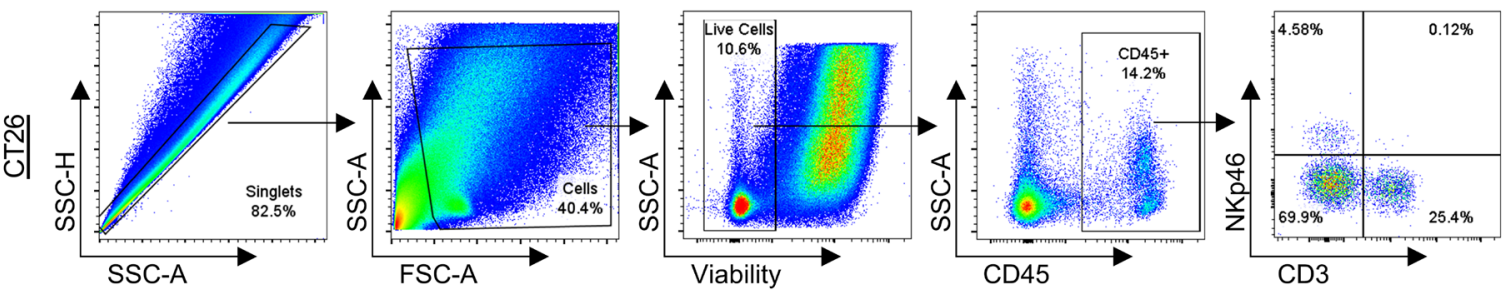

B

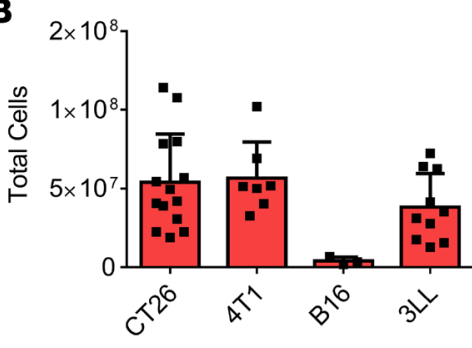

E

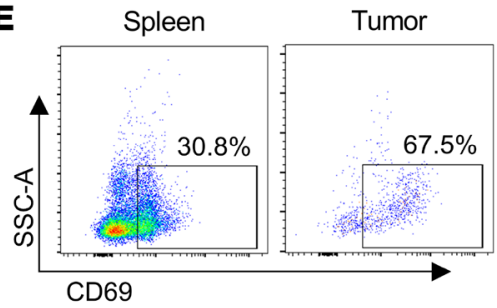

C

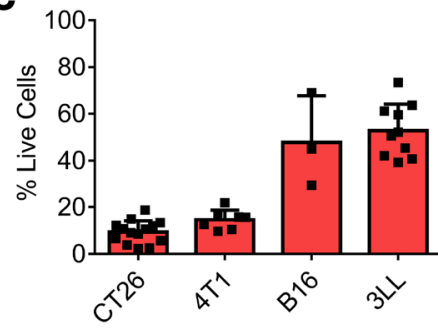

D

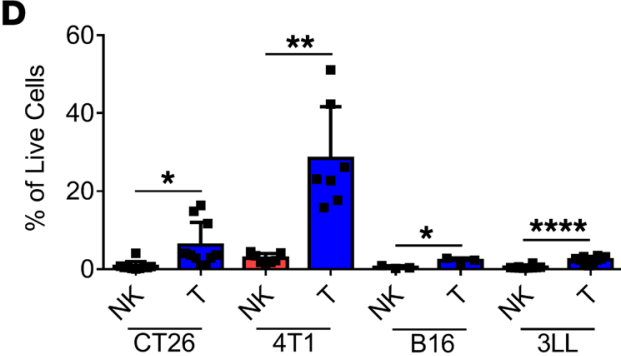

G
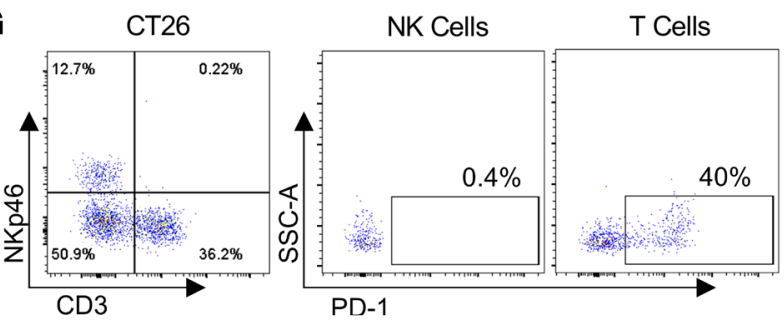

RNA Sequencing

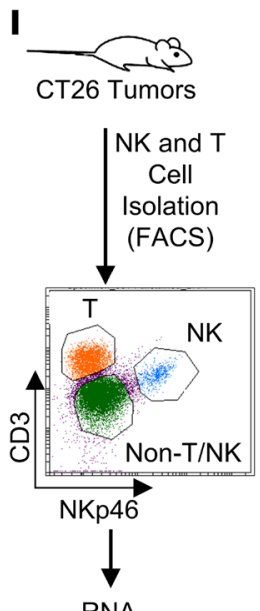

RNA

Sequencing

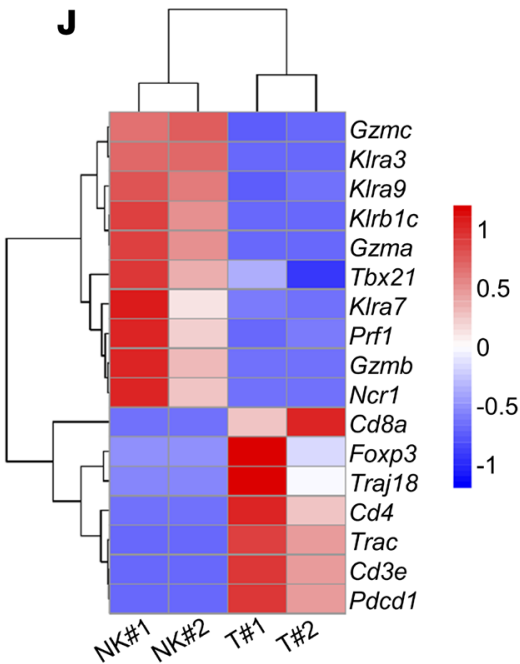

F Spleen
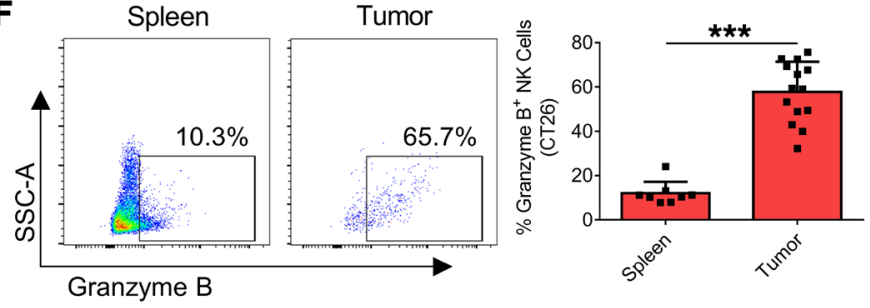

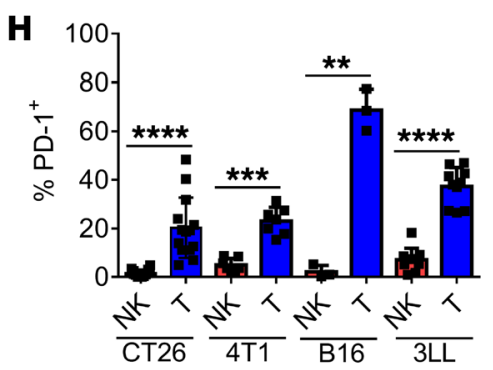

CT26 in PD-1 Transgenic Reporter Mice

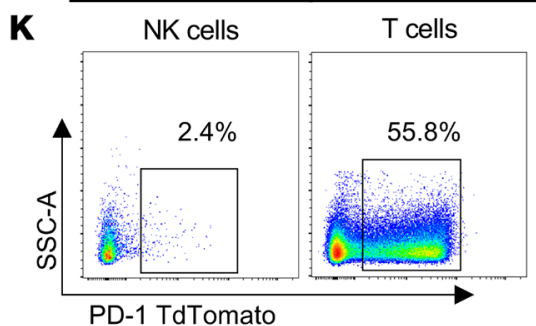

$\mathbf{L}$

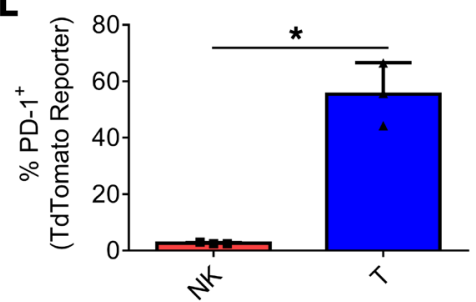


Figure 2. Mouse intratumoral NK cells do not upregulate PD-1. (A) Gating strategy for analysis of intratumoral NK and T cells from CT26 tumors in BALB/c mice. (B) Total cells present, (C) percentage of live cells in tumor, (D) and percentage of live cells in tumor represented by NK and T cells. (E and F) NK cells in CT26 tumors show significantly increased expression of activation marker CD69 and functional marker granzyme B compared with splenic NK cells. (C) Representative NK cell (CD3-NKp46+) and T cell (CD3+NK1.1-) parent gating shows the expression of PD-1 on NK and T cells for CT26 SC tumors. (H) Aggregate expression across tumor models shows consistently minimal expression of PD-1 on NK cells, with significantly higher expression on T cells. (I) Schematic for generating and isolating intratumoral NK and T cells from CT26 tumor-bearing BALB/c mice and (J) RNA-Seq analysis of intratumoral NK and T cells. (K) PD-1 reporter mice were implanted with SC CT26 tumors and analyzed for PD-1 expression. Using the reporter system, PD-1 expression on intratumoral NK cells remains minimal compared with that in T cells, as seen by representative flow plots and (L) summary data. (A-H) Data are shown as mean \pm SD for $n=3-14$ mice/group and are representative of 2 to 3 independent experiments. (I and J) Tumors pooled from $n=4$ (no. 1) and $n=6$ (no. 2) mice/ group. ( $K$ and $\mathbf{L}$ ) Data are shown as mean \pm SD for $n=3$ mice/group. ${ }^{*} P<0.05$; ${ }^{* *} P<0.01$; ${ }^{* *} P<0.001$; ${ }^{* * *} P<0.0001$, paired Student's $t$ test.

in CT26 intratumoral NK cells, while expression was elevated in intratumoral $\mathrm{T}$ cells from the same tumors (Figure 2J). We then analyzed PD-1 expression in NK cells from CT26-bearing transgenic BALB/c mice genetically engineered to express a TdTomato reporter when PD-1 is expressed (Supplemental Figure 3, D and E). PD-1 expression on intratumoral NK cells remained minimal, especially when compared with the robust PD-1 expression on intratumoral $\mathrm{T}$ cells (Figure 2, $\mathrm{K}$ and $\mathrm{L}$ ). An additional benefit of the PD-1 reporter mouse is the ability to detect any expression of PD-1 (regardless of intracellular or extracellular protein location), which confirmed minimal expression of PD-1 in NK cells. Thus, using antibody-based flow cytometry involving different hybridoma clones to PD-1, RNA-Seq, and transgenic fluorescent PD-1 reporter mice, we observed minimal PD-1 expression on tumor-infiltrating NK cells, especially when compared with robust PD-1 expression on intratumoral $\mathrm{T}$ cells.

In vivo acute $M C M V$ infection of mice leads to marked NK cell activation and upregulation of TIGIT but not PD-1. The early response to cytomegalovirus (CMV) infection is dominated by NK cells, making MCMV an ideal model for evaluating the expression and role of PD-1 on highly activated NK cells (37-39). Recent reports indicate that PD-1 can be detected on NK cells at 72 hours following infection (24). Therefore, we evaluated WT and Rag2 NK cells in vivo following MCMV infection. Spleen and liver tissues were analyzed 72 hours after inoculation during peak infection (Figure 3A). Infection was confirmed by viral titers in the liver (Figure 3B). Infected mice also had serum corticosterone concentrations of 200 to $300 \mathrm{ng} / \mathrm{mL} 48$ hours after infection, which has been previously implicated in promoting PD-1 expression on NK cells after infection (ref. 24 and Supplemental Figure 5A). Distinct $\mathrm{NK}\left(\mathrm{CD} 3^{-} \mathrm{NK} 1.1^{+}\right)$and $\mathrm{T}\left(\mathrm{CD}^{+} \mathrm{NK} 1.1^{-}\right)$cell populations were identified within uninfected and infected WT mice (Figure $3 \mathrm{C}$ and Supplemental Figure 5B). MCMV-infected mice exhibited significant and marked increases in the percentages of expression of activation markers CD69 (88\% $\pm 5 \%$ vs. $10 \% \pm 3 \%, P<0.0001)$ (Figure 3D) and Thy1.2 (77\% $\pm 2 \%$ vs. $34 \% \pm 3 \%, P<0.0001)$ in both the spleen and liver (Figure 3E and Supplemental Figure 5C).
Under these highly activated conditions, we again observed minimal PD-1 expression on splenic NK cells $(0.5 \% \pm 0.3 \%$ vs. $1.4 \% \pm$ $0.06 \%, P=0.0099$ ) (Figure $3, \mathrm{~F}$ and $\mathrm{H}$ ) and liver NK cells (Supplemental Figure 5, C and D). However, in contrast, we detected marked increases in expression of the immunoregulatory marker TIGIT on NK cells in both the spleen $(3.1 \% \pm 1.3 \%$ resting vs. $27.7 \%$ $\pm 7.5 \%$ after MCMV, $P=0.0049$ ) (Figure $3, \mathrm{G}$ and $\mathrm{H}$ ) and the liver (Supplemental Figure 5, C and D). Similar results were observed using T cell-deficient Rag2- mice with higher expression of CD69 on CD3-NK1.1 ${ }^{+}{ }^{-N K p} 46^{+}$NK cells $(90.3 \% \pm 0.7 \%)$ (Figure 3I) and consistently minimal expression of PD-1 on NK cells in the spleen $(1.2 \% \pm 0.2 \%)$ (Figure $3 \mathrm{~J})$ and the liver $(1.2 \% \pm 0.02 \%)$ following infection (Supplemental Figure 5, C and D). In contrast, TIGIT was significantly upregulated on activated NK cells from both the spleen $(25.5 \% \pm 1.7 \%)$ and the liver $(48.6 \% \pm 2.6 \%)$ (Figure $3 \mathrm{~J}$ and Supplemental Figure 5, C and D). By qRT-PCR, splenocytes from infected $\mathrm{Rag}_{2}{ }^{--}$mice demonstrated near absent transcript expression of PD-1 after MCMV, while functional marker granzyme B was markedly upregulated $(\sim 15$-fold, $P<0.0001)$ (Figure $3 \mathrm{~K})$. At day 7 after infection in WT mice (Figure 3L), we observed increased PD- 1 expression only on T cells $(1.1 \% \pm 0.6 \%$ vs. $25.2 \%$ $\pm 11.8 \%, P=0.0002$, Figure $3 \mathrm{M}$ ), regardless of the PD-1-specific hybridoma used (Supplemental Figure 4C). Overall, we observed significant expression of PD-1 on splenic T cells 5-7 days after MCMV infection compared with negligible expression on splenic NK cells (Figure 3N).

Anti-ASGM1 depletes activated PD-1 ${ }^{+} T$ cells following MCMV infection. In addition to the subjective nature of flow cytometry gating strategies, commonly used in vivo NK depletion strategies pose challenges when attempting to attribute NK-specific effects. This is in part due to the lack of truly NK-specific depleting antibodies in mice. Administration of a polyclonal anti-serum to the marker anti-asialo-GM1 ( $\alpha$ ASGM1) has been commonly used to ascribe NK cell-specific effects, particularly in mice lacking the NK marker NK1.1, which is not present in the BALB/c strain. However, ASGM1 is known to be expressed on multiple cell types, particularly during activation or inflammatory states, and there have been reports that ASGM1-based depletion has effects on non-NK cellular constituents, including activated T cells (40-44). We evaluated the expression of ASGM1 and the impact of ASGM1 depletion following acute MCMV infection (Figure 4A). Total NK cells $\left(\mathrm{CD}^{-}{ }^{-N K p} 46^{+}\right)$were significantly decreased with ASGM1 depletion in mice either with or without MCMV infection (Figure 4, B and C, and Supplemental Figure 6A). However, compared with those in untreated mice, total $\mathrm{T}$ cells in the spleen were significantly depleted when $\alpha$ ASGM1 was administered during acute MCMV infection (Figure 4C). We also observed a partial decrease in the frequency of T cells in the spleen after using $\alpha$ NK1.1 (PK136) in MCMV-infected (but not resting) mice (Supplemental Figure 6, A and B). Representative flow cytometry staining for ASGM1 as a cell-surface marker on $\mathrm{T}$ cells also showed that ASGM1 significantly increased with MCMV infection ( $16 \% \pm 1 \%$ untreated vs. $35 \% \pm 5 \%$ with MCMV, $P=0.0189$ ) (Figure $4 \mathrm{D}$ ), and ASGM1 ${ }^{+} \mathrm{T}$ cells were significantly decreased after $\alpha$ ASGM1 depletion compared with MCMV infection alone (Figure 4E). Importantly, PD-1 expression was significantly increased along with ASGM1 following MCMV infection on splenic T cells $(9 \% \pm 2 \%$ untreated vs. 
A C57BL/6 or Rag2--

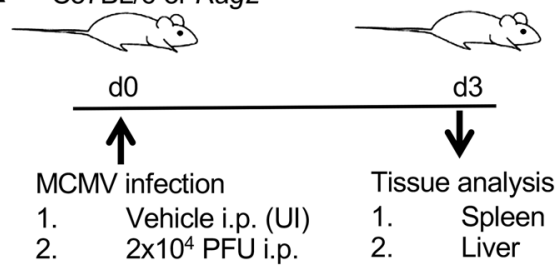

B

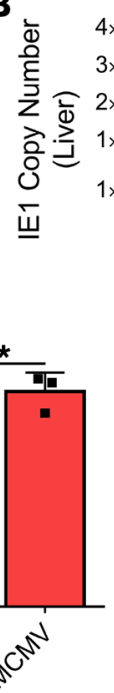

D

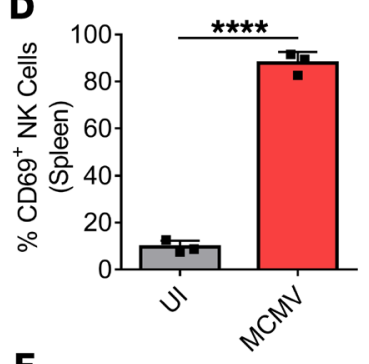

$\mathbf{F}$

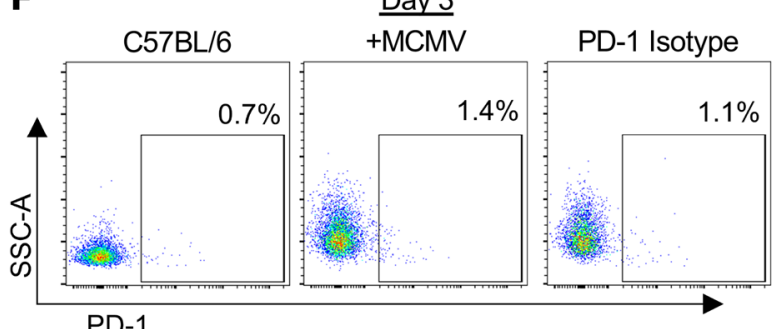

H

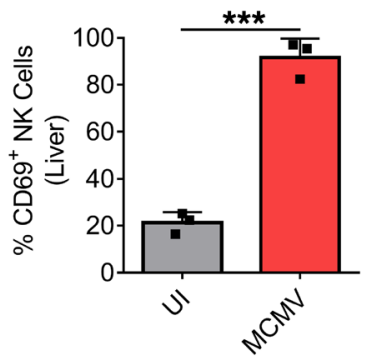

$\underline{\text { Day } 3}$

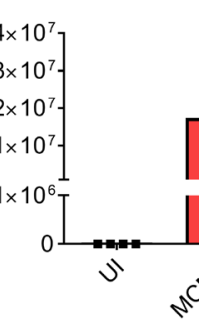

E

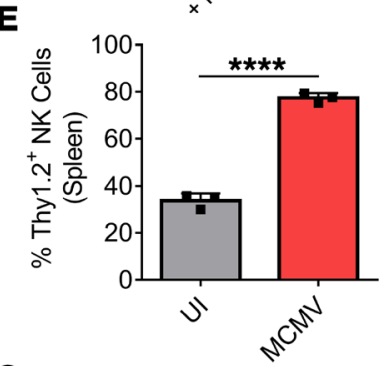

G

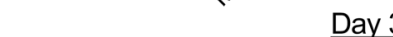

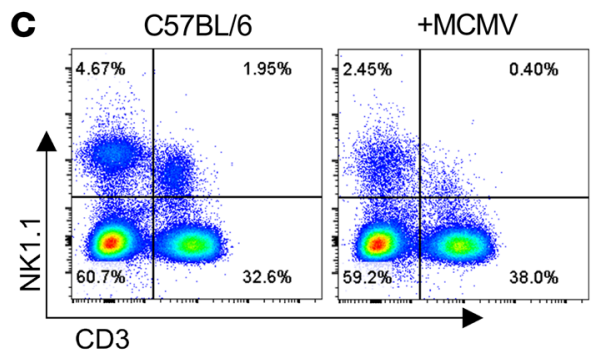

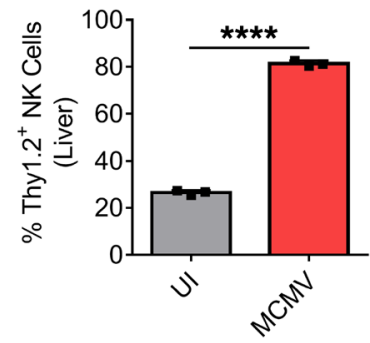

Day 3

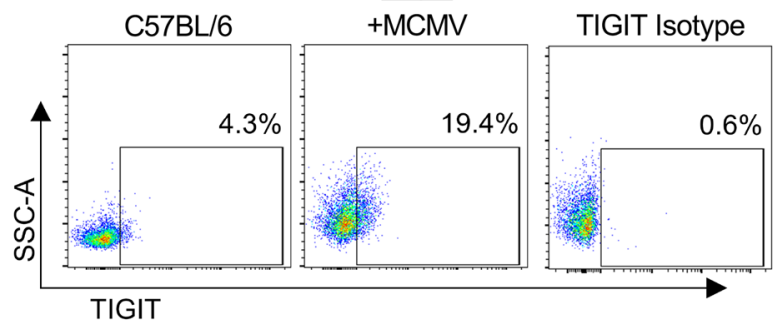

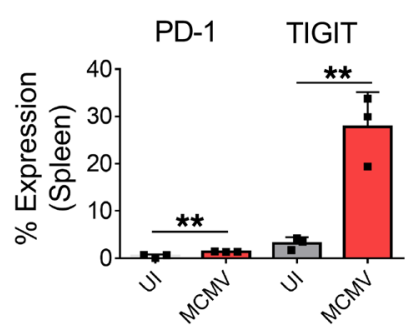

I

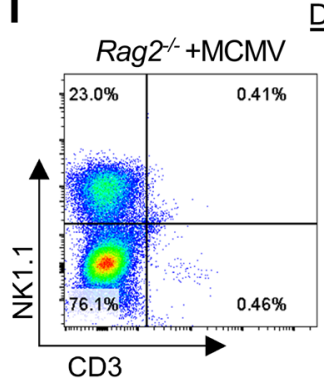

$\mathbf{L}$
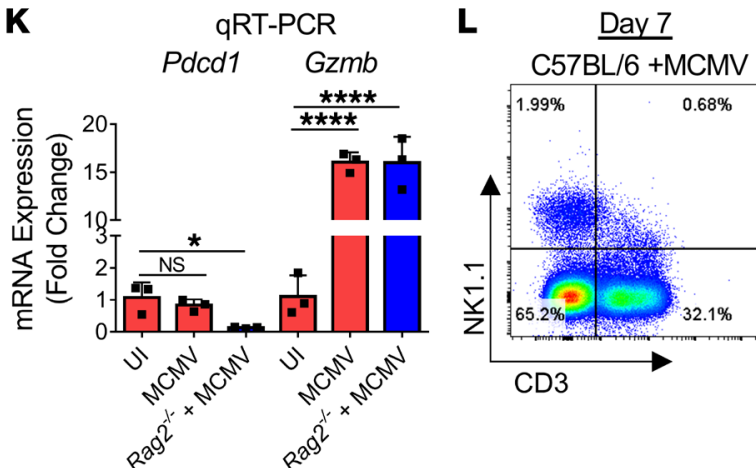

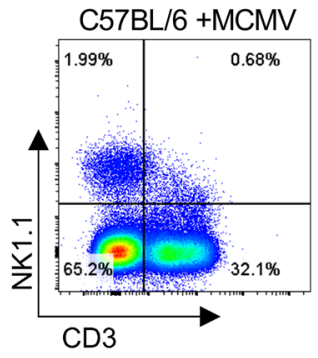

J

Day 3

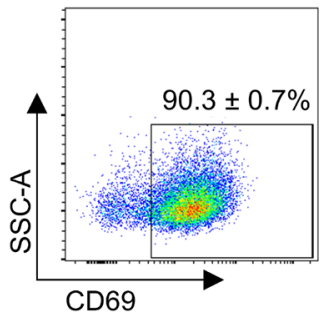

M
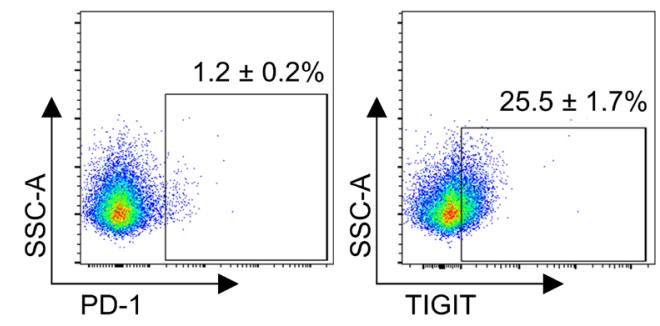

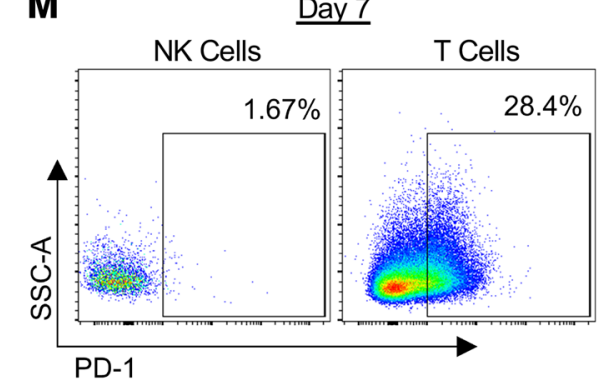

$\mathbf{N}$

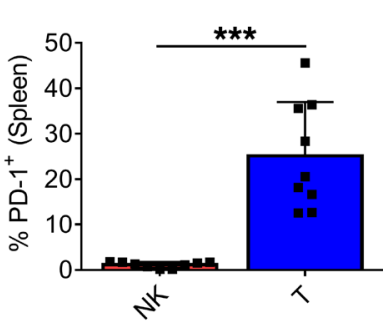

Figure 3. In vivo acute MCMV infection leads to upregulation of TIGIT, not PD-1, on highly activated NK cells. (A) WT C57BL/6 and Rag2 $2^{-1-}$ mice were infected with $2 \times 10^{4}$ PFU MCMV. (B) At 72 hours, viral titers were elevated in the liver. (C) Representative parent gating from spleens of mice shows NK (CD3-NK1.1 $1^{+}$and T (CD3+NK1.1-) cell populations. NK cells were further gated on NKp46 and compared with those of uninfected mice. MCMV-infected mice had significant upregulation of (D) CD69 and (E) Thy1.2 on NK cells in the spleen and liver at 3 days after infection. (F) PD-1 expression on NK cells in WT mice remained minimal, while (C) TIGIT was upregulated. (H) Though minimal (1\%-2\% increase), the measured increase in PD- 1 was statistically significant, while TIGIT was upregulated by $15 \%$ to $25 \%$ under identical conditions. (I) Parent gating of splenocytes from acute MCMV infection in Rag $2^{-/-}$mice shows marked upregulation of CD69, while (J) PD-1 was minimal and TIGIT upregulation significant. (K) PD-1 mRNA expression on MCMV-infected Rag2 ${ }^{-/-}$splenocytes was minimal compared with uninfected WT splenocytes, while both infected groups exhibited marked upregulation of granzyme B. (L) At day 7 after infection, (M) PD-1 expression on T cells was upregulated, while NK expression of PD-1 remained minimal. (N) Summary data of PD-1 expression on NK and T cells at 5 to 7 days after infection. Data are shown as mean $\pm S D$ for $n=3$ mice/group and are representative of 3 independent experiments (B, D, E, H, and $\mathbf{K})$. Data are shown as mean $\pm \mathrm{SD}$ for $n=2-3$ mice/group, showing results from 4 independent experiments using 1 to $2 \times 10^{4} P F U$ MCMV $(\mathbf{N})$. ${ }^{* *} P<0.01 ;{ }^{* *} P<0.001$; ${ }^{* * *} P<0.0001$, unpaired Student's $t$ test $(\mathbf{D}, \mathbf{E}$, and $\mathbf{H})$ and 1-way ANOVA compared with uninfected splenocytes $(\mathbf{K})$. 
A

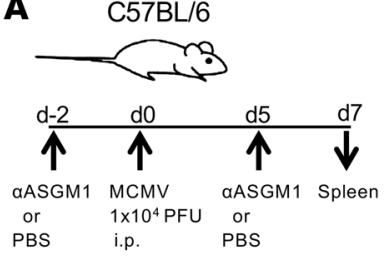

B

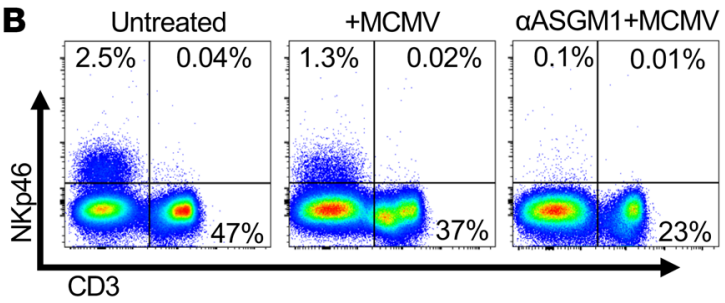

D

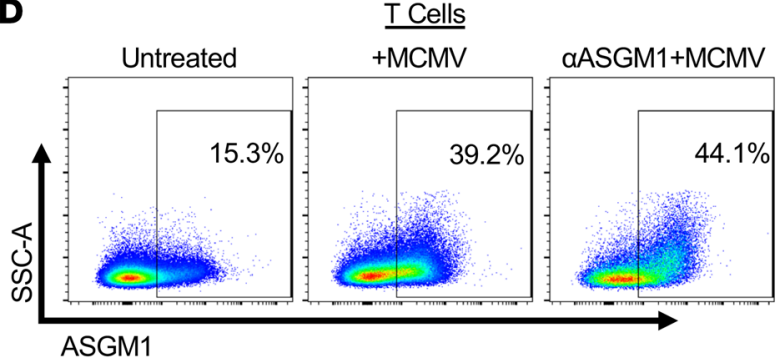

$\mathbf{F}$

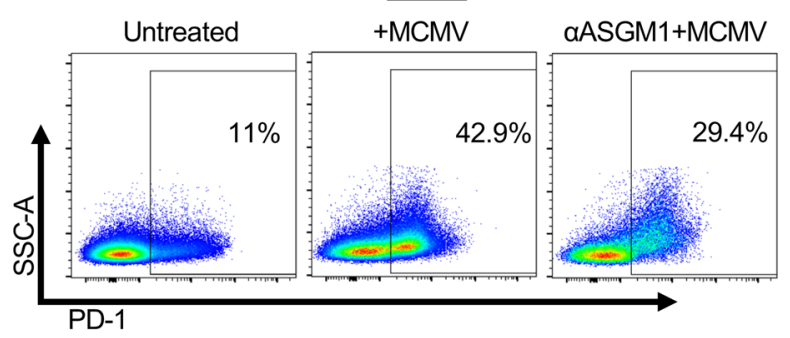

H

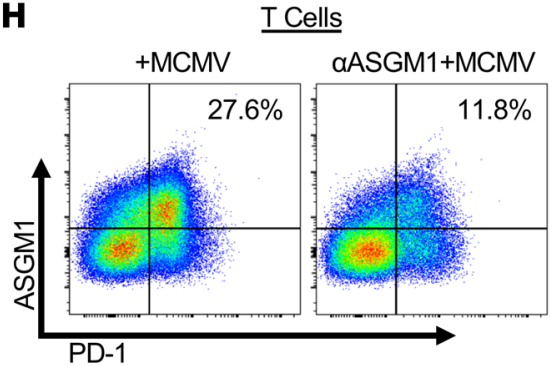

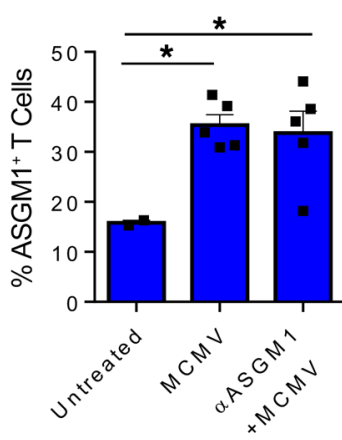
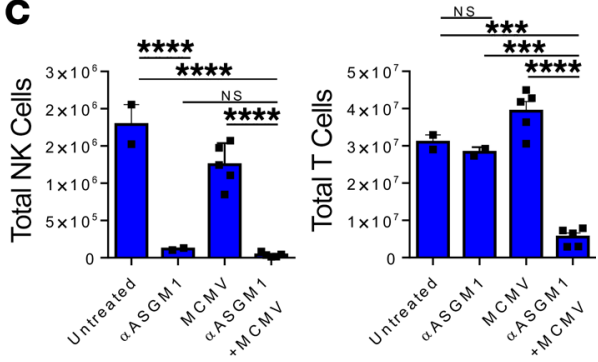

$E$

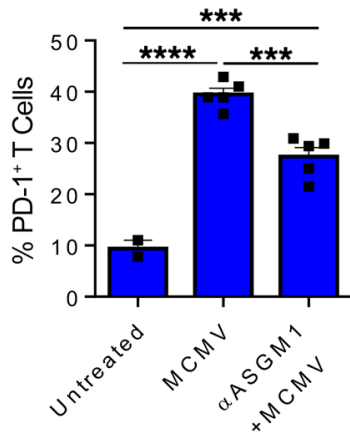

G
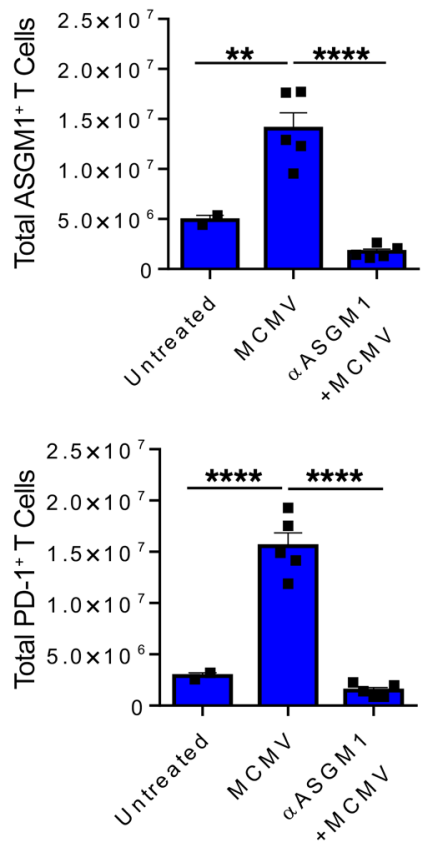

Figure 4. Anti-ASCM1 depletes activated PD-1+ $T$ cells following MCMV infection. (A) Schematic depicts NK depletion strategies and MCMV infection in C57BL/6 mice. (B) Representative parent gating is shown in untreated, MCMV-infected, and MCMV-infected mice with ASGM1 depletion. (C) Total NK and T cells were collected from the spleens showing significant loss of NK cells with $\alpha A S G M 1$ and MCMV+ $\alpha A S G M 1$ and significant loss of T cells with MCMV+ $\alpha$ ASCM1 only. (D) Representative ASGM1 expression on T cells shows increase in ASCM1 expression with MCMV-induced T cell activation. (E) Mean percentage of ASCM1 ${ }^{+}$T cells was significantly higher in the MCMV-infected groups, while total ASCM1+ $T$ cell numbers were significantly lower following MCMV+ $\alpha$ ASGM1. (F) Representative PD-1 expression on T cells shows increase in PD-1 expression with MCMV-induced T cell activation. (C) Mean percentage of PD-1+ $\mathrm{T}$ cells was significantly higher in the MCMV-infected groups, while total PD- $1^{+} T$ cells were significantly lower following MCMV+ $\alpha A S C M 1$. (H) Representative gating shows the proportion of ASGM1+PD-1+ $\mathrm{T}$ cells in the MCMV-infected groups and the impact of ASGM1 depletion on reducing activated PD-1+ T cells after MCMV infection. Data are shown as mean $\pm S D$ for $n=2-5$ mice/group and are representative of 2 independent experiments. ${ }^{*} P<0.05$; ${ }^{* *} P<0.01$; ${ }^{* *} P<0.001$; ${ }^{* * *} P<0.0001$, 1-way ANOVA with multiple group comparisons.

$39 \% \pm 3 \%$ with MCMV, $P<0.0001$ ) (Figure $4 \mathrm{~F}$ ), and significant decreases in the frequency (Figure $4 \mathrm{~F}$ ) and total numbers (Figure $4 \mathrm{G}$ ) of PD- $1^{+} \mathrm{T}$ cells were evident in the spleens of mice after MCMV infection and $\alpha$ ASGM1 (Figure $4 \mathrm{H}$ ), an effect we did not observe with NK1.1 depletion in MCMV infection (Supplemental Figure 6C). These results indicate that, following acute viral infection, both PD-1 and ASGM1 are upregulated on activated T cells, whereas NK cell expression of PD-1 remains minimal.
In vitro activation of human $P B M C$ leads to $P D-1$ expression on $T$ cells, not NK cells. Although we were unable to demonstrate significant expression of PD-1 on murine NK cells either in vitro or in vivo, reports have also shown PD-1 expression on human NK cells (23). Therefore, we next evaluated PD-1 expression on human $\mathrm{NK}$ and $\mathrm{T}$ cells, starting with various in vitro activation strategies. Following culture of PBMCs for 3 days in media only (Figure 5A), "resting" human NK cells $\left(\mathrm{CD}^{-}{ }^{-} \mathrm{CD} 56^{+}\right)$and T cells $\left(\mathrm{CD}^{+}{ }^{+} \mathrm{CD} 56^{-}\right)$ 
A

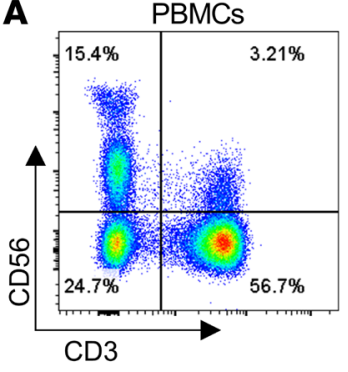

D + ConA $2.5 \mu \mathrm{g} / \mathrm{mL}$

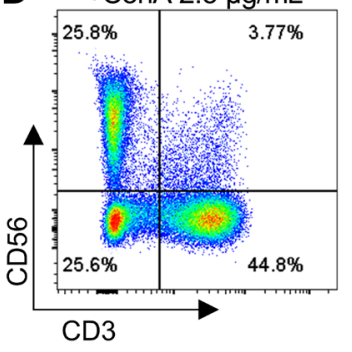

G $\quad+\mathrm{IL}-21000 \mathrm{IU} / \mathrm{mL}$

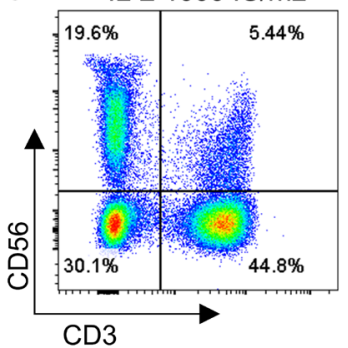

J +lL-15 $100 \mathrm{ng} / \mathrm{mL}$

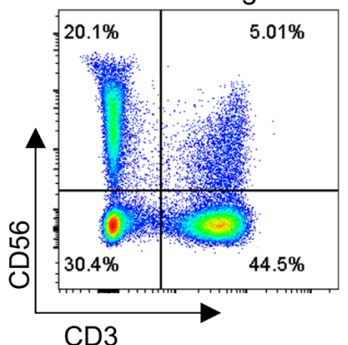
CD3
B

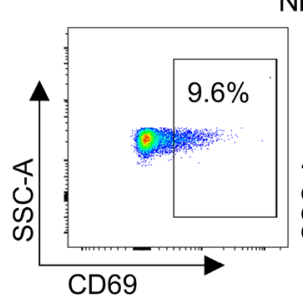

E

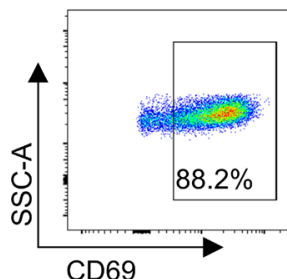

NK Cells

H

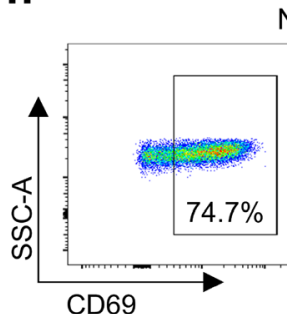

NK Cells

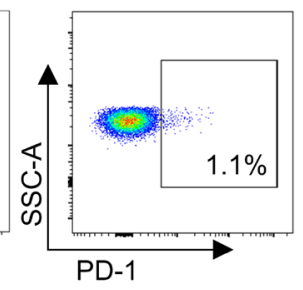

$\mathbf{K}$

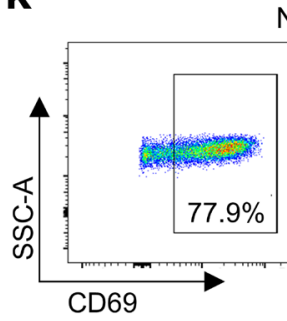

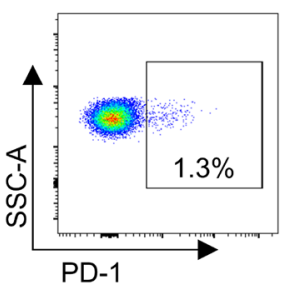

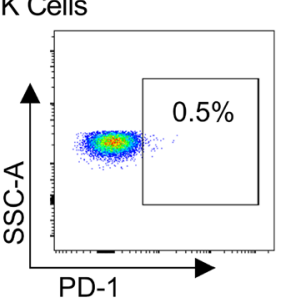

PD-1
NK Cells

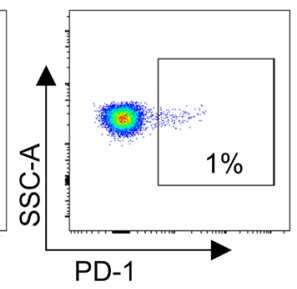

C

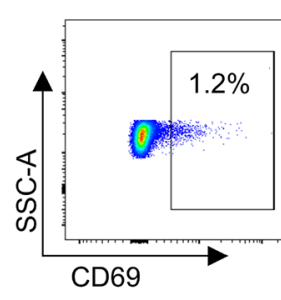

$\mathbf{F}$

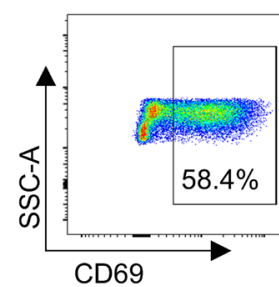

T Cells

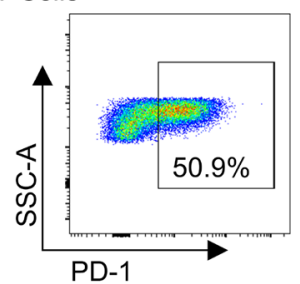

T Cells

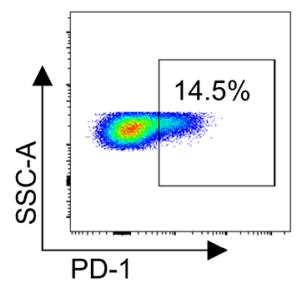

I

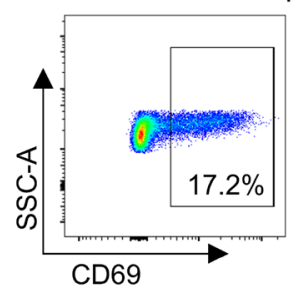

T Cells

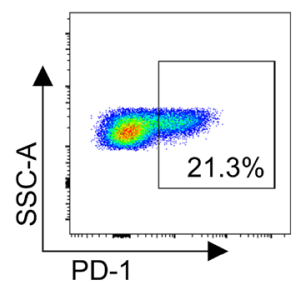

M

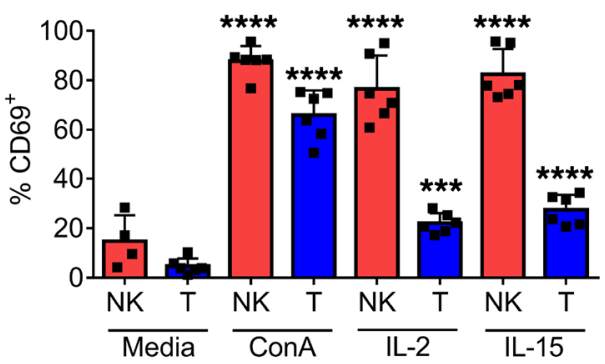

$\mathbf{L}$

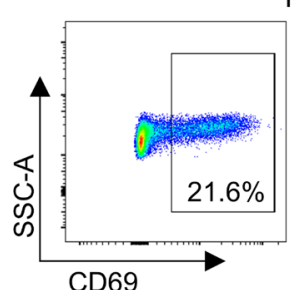

T Cells

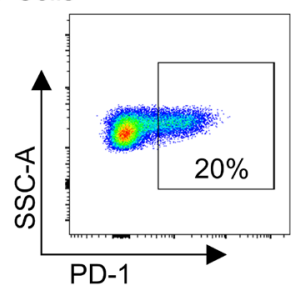

$\mathbf{N}$

PD-1 Expression

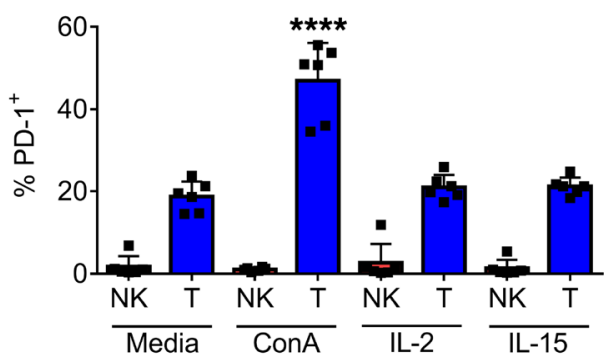

Figure 5. In vitro activation of human PBMCs leads to PD-1 expression on T cells, not NK cells. (A) Representative gating shows NK and T cell populations isolated from human PBMCs after 72 hours in media. (B) Resting CD3-CD56 ${ }^{+}$NK cells lack expression of activation marker CD69 and are negative for PD-1, (C) while resting $\mathrm{CD}^{+}{ }^{+} \mathrm{CD56}-\mathrm{T}$ cells also lack expression of $\mathrm{CD69}$, but show baseline expression of PD-1. (D) After culture in ConA for 72 hours, NK cells show increased CD56 expression, (E) with upregulation of CD69 and continued absence of PD-1. (F) After ConA stimulation, T cells exhibit increased CD69 expression and robust PD-1 expression. (G) After culture in $1000 \mathrm{IU} / \mathrm{mL}$ rhIL-2 for 72 hours, (H) NK cells upregulate CD69 with minimal PD-1 expression. (I) In contrast, CD3 ${ }^{+}$ T cells exhibit moderate increases in both CD69 and PD-1. (J) After culture in 100 ng/mL rhlL-15 for 72 hours, (K) NK cells upregulate CD69 with minimal PD-1 expression. (L) Similarly to what occurs with IL-2 stimulation, CD3 ${ }^{+}$T cells exhibit moderate increases in both CD69 and PD-1 after IL-15 stimulation. (M) Summary data show significantly increased expression of CD69 on NK and T cells with either ConA, IL-2, or IL-15 compared with media alone. (N) Under identical conditions, PD-1 was significantly upregulated on ConA-stimulated T cells with no change in PD-1 expression on NK cells under any conditions. Data are shown as mean \pm SD for $n=4-6$ unique human donors. ${ }^{* *} P<0.001$; ${ }^{* * *} P<0.0001,1$-way ANOVA compared with cell-specific media only controls. 
expressed low levels of activation marker CD69, and NK cells were negative for PD-1 (Figure 5, B and C). Conversely, T cell expression was variable, as shown in Figure 5C. After 72 hours of ConA stimulation (Figure 5D), both $\mathrm{NK}$ and $\mathrm{T}$ cells markedly increased expression of CD69, while only T cells had significantly increased PD-1 expression $(19 \% \pm 4 \%$ in media vs. $47 \% \pm 9 \%$ in ConA, $P<$ 0.0001 ) (Figure 5, E and F). Following 72 hours of culture in 1000 IU/mL rhIL-2 (Figure 5G), NK cells increased percentage of CD69 expression, but PD-1 expression remained limited to T cells $(3 \% \pm$ $5 \%$ on NK vs. $21 \% \pm 3 \%$ on T cells, $P<0.0001$ ) (Figure $5, \mathrm{H}$ and I). We also examined the effect of rhIL-15 given evolving data for IL-15-mediated function and induction of NK cell exhaustion (45, 46), and similar results were obtained compared with IL-2 (Figure $5, \mathrm{~J}-\mathrm{L}$ ). A summary of data from all human donors is presented for CD69 (Figure 5M) and PD-1 (Figure 5N). We also examined the well-characterized NK92 cell line (47), which has been reported to have variable expression of PD-1 $(12,48)$. After culture in 500 IU/mL rhIL-2, we detected high expression of TIGIT, but minimal PD-1 expression, as determined by both flow cytometry and qRTPCR (Supplemental Figure 7).

Ex vivo generation of expanded and activated human NK cells upregulates TIGIT but not PD-1. With the hypothesis that cytokine alone may not be a sufficient stimulus to induce PD-1 expression on NK cells, we next analyzed isolated human NK cells for PD-1 expression using coculture with a human leukemia cell line transduced with NK costimulatory molecules (K562 clone 9-mb2141BBL) and rhIL-2 $(16,49,50)$. Purified human NK cells were analyzed over the course of 21 days for expression of phenotypic and functional markers (Figure 6, A and B). After culture, mRNA expression of Ki67 was significantly increased at all time points (Figure 6C). These results were confirmed by flow cytometry (Figure 6D and Supplemental Figure 8A). We detected a 10-fold expansion of NK numbers by day 7, 100-fold expansion by day 12, and approximately 1000-fold expansion by day 19 (Figure 6E). Similarly to what was seen in our murine data, human NK cells markedly and significantly upregulated CD69 (Figure 6F), granzyme B (Figure 6G), and degranulation marker CD107a (Figure 6H). Yet PD-1 expression was again minimal ( $\leq 2 \%$ at all time points, as shown in Figure 6I, gated using isotype controls; Supplemental Figure 8, B and C). Additionally, PD-1 expression was minimal by qRT-PCR (Figure 6J). In contrast to minimal PD-1 expression, we observed significant upregulation of TIGIT expression $(20 \% \pm$ $11 \%$ at day 0 vs. $62 \% \pm 13 \%$ at day $6,77 \% \pm 5 \%$ at day $12,87 \% \pm 3 \%$ at day $21, P<0.001$ for all time points compared with day 0) (Figure $6 \mathrm{~K}$ ), confirmed by mRNA analysis (Figure 6L). We then added $100 \mathrm{ng} / \mathrm{mL}$ rhIL-15 to our standard feeder line and rhIL-2 coculture conditions, as prolonged rhIL-15 exposure has been shown to induce PD-1 expression on human NK cells in vitro (23). At day 6 and day 11 under these conditions (Supplemental Figure 8D), we observed minimal PD-1 expression (Supplemental Figure 8E). As recent studies have also shown that dogs provide a link in cancer immunotherapy studies, with the potential for speeding translation of NK immunotherapy $(51,52)$, we performed a similar expansion of dog NK cells with feeder line coculture and rhIL-2 and used qRT-PCR at days 14 and 21. Using canine-specific markers, PCR analysis revealed upregulation of NK-specific marker NKp46 with concomitant increases in Ki67, TIM-3, and TIGIT. Importantly, as with ex vivo expansion of murine and human NK cells, we did not detect significant expression or upregulation of PD-1 on dog NK cells by qRT-PCR (Supplemental Figure $8 \mathrm{~F}$ ) despite the marked expansion of highly activated canine NK cells demonstrating consistency of the phenotype across species.

Tumor-infiltrating NK cells from human tumors show increased TIGIT and minimal PD-1 expression. Although prior reports have identified PD-1 expression on circulating and intratumoral NK cells in human cancer patients, these studies have reported wide variation and have been inconsistent depending on tumor type $(21,53,54)$. We set out to investigate not only PD-1 expression on the NK and T cell repertoire from primary surgical specimens, but also the absolute number of $\mathrm{NK}$ and $\mathrm{T}$ cells present in tumors to provide a context on the extent and magnitude of these populations. We chose to focus on soft tissue sarcomas, colon cancer, and head and neck squamous cell carcinomas (HNSCC), as preclinical and clinical studies have suggested these tumors are NK responsive (55-58). As shown in Figure 7A, preoperative CT and PET/ CT scans show size and location of patient tumors. H\&E staining demonstrates representative morphology from individual specimens (Figure 7B). Representative flow cytometry shows NK and $\mathrm{T}$ cell populations from blood (Figure 7C) and tumor (Figure 7D and Supplemental Figure 9A), including in patient SA-1338. Circulating NK cells expressed minimal PD-1, but prominent TIGIT expression, whereas circulating $\mathrm{T}$ cells expressed both PD-1 and TIGIT (Figure 7C). Within the tumor of the same patient (Figure 7D), we observed a markedly greater infiltrate of T cells compared with NK cells. In addition, the intratumoral NK infiltrate showed a predominance of CD56 $6^{\mathrm{dim}} \mathrm{NK}$ cells in the tumor compared with the blood. Notably, intratumoral human NK cells expressed minimal PD-1 (1.1\%), in contrast with the high percentage of PD-1 expression on intratumoral T cells (47.3\%). Interestingly, TIGIT expression was present on both NK and T cells (Figure 7D). We also analyzed PBMCs and tumor-infiltrating lymphocytes (TILs) from a colon cancer patient (Figure 7, E and F). Within the peripheral blood, the distribution of NK and T cells was comparable to that of sarcoma patients, and we observed similar immune phenotype with minimal percentage of expression of PD-1 on NK cells (2.9\% PBMCs, $0.2 \%$ tumor), but positive PD-1 expression on $\mathrm{T}$ cells (15.8\% PBMCs, $26.6 \%$ tumor). We again identified notable expression of TIGIT on both peripheral NK and T cells, although as a rule, TIGIT expression was higher on the NK cell population compared with T cells (Figure 7E). Interestingly, in some patients, we observed a higher proportion of NK cells in the tumor microenvironment (TME), but PD-1 expression on NK cells remained minimal (0.25\%), while TIGIT expression was elevated on both NK and T cells (Figure 7F). We then analyzed patients with HNSCC, as NK cells have been shown to be active in these cancers (59). Supplemental Figure 9B shows a preoperative PET/CT. Similarly to what was seen in the other cancer patients, PD-1 expression was minimal on blood NK cells (0.9\%) compared with T cells $(16.4 \%)$ (Supplemental Figure 9C). Within the tumor, both NK and T cells had increased expression of CD69, while PD-1 expression on intratumoral NK was minimal at 5.1\% compared with $78.6 \%$ on $\mathrm{T}$ cells. In this patient, intratumoral NK and T cells had comparable TIGIT expression (Supplemental Figure 9D). We then quantitated the overall numbers and percentages of immune infiltrate in 
A Generation of Expanded Human NK Cells

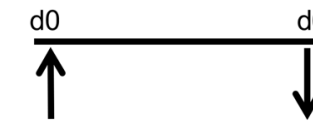

Purified human NK cells K562.Cl9 feeder cell line $100 \mathrm{IU} / \mathrm{mL} \mathrm{IL}-2$

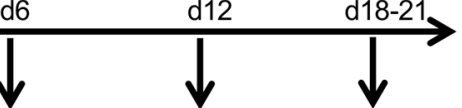

Cell analysis

1. Flow cytometry

2. $\mathrm{qRT}$-PCR
B

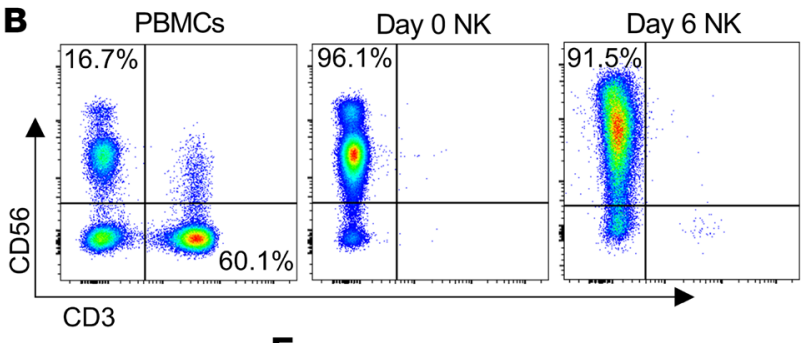

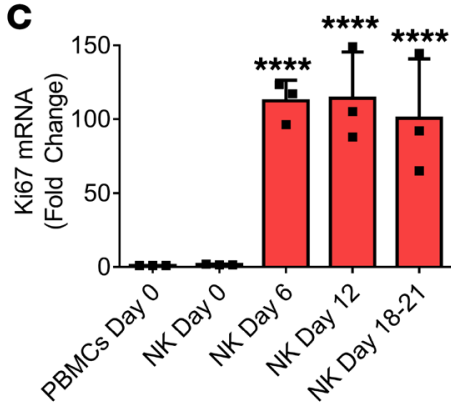

$\mathbf{F}$

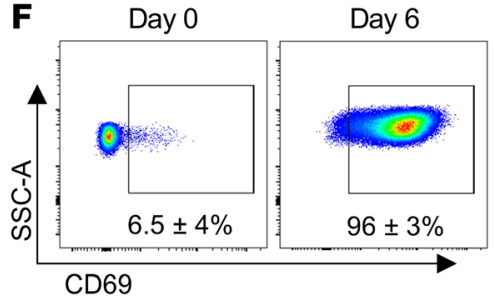

I

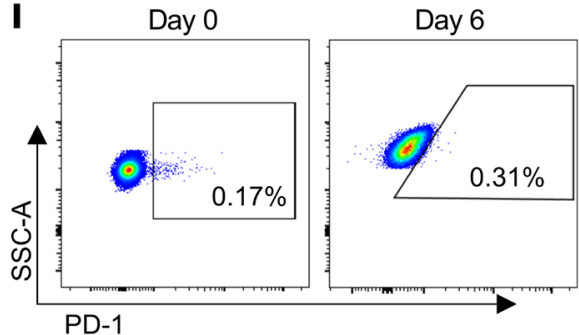

PD-1

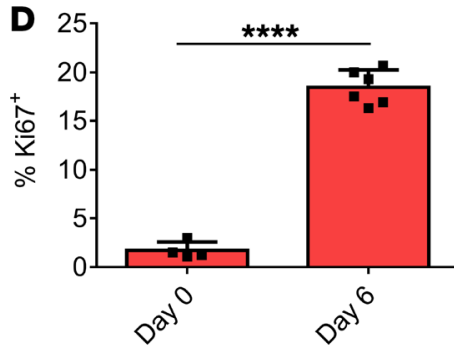

G
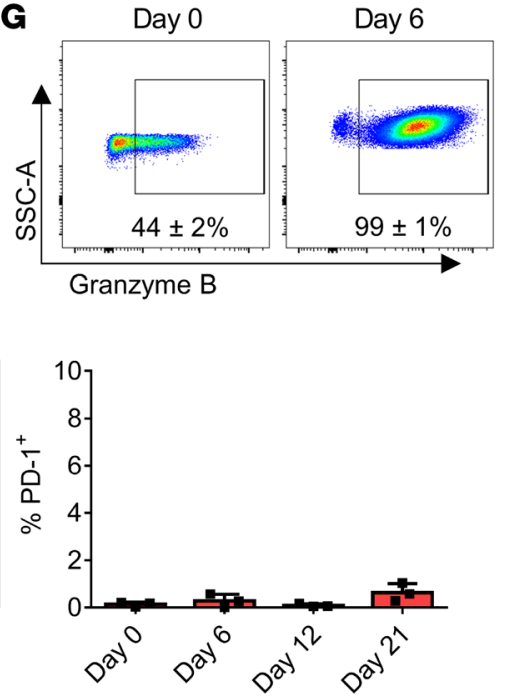

E

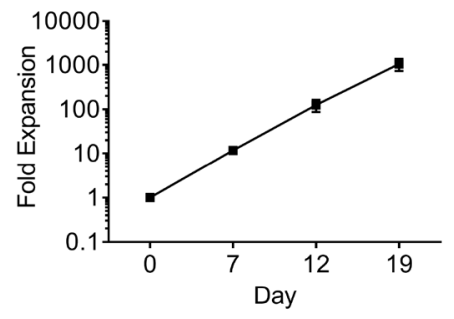

H

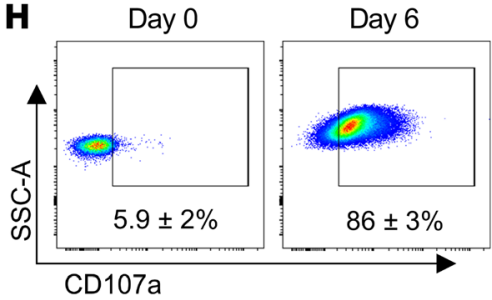

J

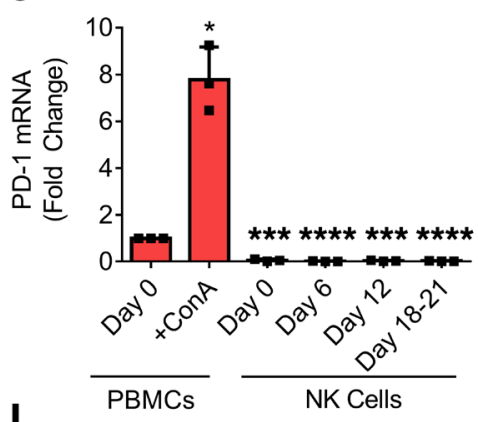

Figure 6. Ex vivo generation of expanded and activated human NK cells upregulates TICIT, not PD-1. (A) Schematic shows method for generating ex vivo human NK cells from healthy donor PBMCs. (B) Representative flow cytometry gating shows NK and T cell populations before and after NK isolation at day 0 and then at day 6 during NK expansion. (C) mRNA expression of Ki67 was significantly increased at all time points, consistent with a highly proliferative state and (D) confirmed by a significant increase in Ki67 $7^{+}$NK cells at day 6 by flow cytometry. (E) Coculture demonstrated robust NK expansion along with significant upregulation of (F) CD69 expression, (G) granzyme B expression, and (H) CD107a degranulation. Although isolated NK cells are highly proliferative, with upregulation of activation and functional markers, (I) there was no expression of PD-1 across all time points analyzed. (J) Isolated NK cells were also analyzed for PD-1 mRNA expression by qRT-PCR and compared with unstimulated (day 0) PBMCs. PD-1 expression on isolated and expanded NK cells was minimal by qRT-PCR at all time points. (K) In contrast, we detected consistent and significant upregulation of TIGIT on NK cells as shown by representative flow cytometry on day 6 and summarized for all time points analyzed. (L) qRT-PCR analysis confirmed significant upregulation of TICIT mRNA expression compared with unstimulated (day 0) PBMCs. Data are shown as mean \pm SD compiled for $n=3(\mathbf{C}$ and $\mathbf{I}-\mathbf{L})$ or $n=2$ (D and E) donors from independent experiments. Data are shown as mean \pm SD for 1 to 2 donors cultured in duplicate or triplicate $(\mathbf{F}-\mathbf{H}) .{ }^{*} P<0.05 ;{ }^{* *} P<0.01 ;{ }^{* *} P<0.001$; ${ }^{* * * *} P<0.0001$, Student's $t$ test (D) or 1-way ANOVA (C and $\left.\mathbf{I}-\mathbf{L}\right)$ compared with day 0 NK (I and $\mathbf{K}$ ) or day 0 PBMCs (J and $\mathbf{L}$ ). 
all tumors represented by TILs and NK and T cells. We observed consistently low numbers of TILs among sarcomas with especially low frequencies and numbers of intratumoral NK cells ( $1 \%$ of total live cells), as shown in Supplemental Figure 9, E and F. Within these small populations, NK cell expression of PD-1 was consistently minimal in both the blood and TME, while T cells exhibited increased expression in the TME compared with blood $(27 \% \pm$ $10 \%$ on peripheral T cells vs. $56 \% \pm 20 \%$ on intratumoral T cells, $P=0.01 ; 1 \% \pm 1 \%$ on peripheral NK cells vs. $4 \% \pm 3 \%$ on intratumoral NK cells, $P=0.1$ ) (Figure $7 G$ ). In contrast, TIGIT was expressed on NK and T cells in the both the blood and the TME (Figure 7H). Overall, PD-1 expression on NK cells was consistently minimal, in marked contrast with TIGIT expression in all TME samples assessed. In contrast, intratumoral $\mathrm{T}$ cell expression of PD-1 was consistently and significantly higher, with T cell populations also expressing TIGIT (Figure 7, I and J).

NK cells activated by hematologic malignancies exhibit minimal PD-1 expression. Since NK cells have a strong correlation with responses in hematologic malignancies (15), we next assessed NK activity and PD-1 expression in vivo in mice using the murine acute myelogenous leukemia cell line C1498. Leukemia-bearing mice (Figure 8A) exhibited multiple liver tumor deposits and enlarged spleens (Figure 8B). As shown in Figure 8, C and D, and Supplemental Figure 10A, in control mice, PD-1 expression in spleen and liver NK cells was nearly absent, and PD-1 expression on T cells was low (spleen, $0.3 \pm 0.07 \%$ vs. $9.7 \pm 1.3 \%, P=0.0002$; liver, 1.0 $\pm 0.4 \%$ vs. $9.1 \pm 4.0 \%, P=0.4$ ). Leukemia-bearing mice showed no significant increase in PD-1 expression on NK cells compared with untreated mice (spleen, $1.5 \pm 1.8 \%$ vs. $0.3 \pm 0.07 \%, P=0.9$; liver, $2.1 \pm 1.6 \%$ vs. $1.0 \pm 0.4 \%, P=0.99)$. However, PD-1 expression on $\mathrm{T}$ cells was upregulated in tumor-bearing mice (Figure 8 , E-G). We then analyzed matched peripheral blood and tumor-infiltrating NK and T cells from a patient with follicular lymphoma (Figure $8 \mathrm{H}$ ). Within the $\mathrm{NK}$ and $\mathrm{T}$ cell populations, expression of CD69 was 55\% and 34\%, respectively, while PD-1 expression was under $1 \%$ on NK cells versus $26 \%$ on T cells. In blood, expression of CD 69 on NK and T cells was $4 \%$ and $1 \%$ respectively, whereas expression of PD- 1 was under $1 \%$ on the NK cells and $23 \%$ on the T cells (Figure 8I and Supplemental Figure 10B). It has been reported that NK cells isolated from patients with hematologic malignancy can be induced to express PD-1 after in vitro culture with rhIL-2 (60). Therefore, we cultured PBMCs from this follicular lymphoma patient for 72 hours in $1000 \mathrm{IU} / \mathrm{mL}$ rhIL-2. Similarly to what was seen in our previous ex vivo culture data, NK cells from this patient showed significantly increased expression of CD69 (99\% $\pm 0.2 \%$ vs. $2 \% \pm 1 \%, P<0.0001), \mathrm{Ki} 67$ (85\% $1 \%$ vs. $1 \% \pm 0.3 \%, P$ $<0.0001)$, and TIGIT ( $87 \% \pm 1 \%$ vs. $36 \% \pm 3 \%, P<0.0001)$ after rhIL-2 stimulation, but there was absence of PD-1 (0.3\% $\pm 0.5 \%$ vs. $0.5 \% \pm 0.2 \%, P=0.2$ ) (Figure $8 \mathrm{~J}$ ). Finally, we evaluated PD-1 expression on healthy NK cells in the context of direct cytotoxicity assays against human leukemia targets (Figure 8K). After coculture with K562 target cells and rhIL-2, CD107a expression reached $72 \%$ $\pm 1 \%(P<0.0001$ compared with media only and rhIL-2). However, PD-1 expression remained minimal on NK cells in all conditions $(2 \% \pm 0.8 \%$ vs. $1.4 \% \pm 0.06 \%$ and $1.6 \% \pm 0.06 \%, P=0.3$ for each comparison, Supplemental Figure 10C). We also observed increased expression of CD69, Ki67, and IFN- $\gamma$ after coculture with
rhIL-2 and K562 targets $(P<0.0001$ for all). Exposure to rhIL-2 and K562 target cells also led to increased expression of TIGIT on NK cells ( $>90 \%$ and $P<0.0001$, Supplemental Figure 10D). These data indicate that, in human cancers, under a variety of conditions, NK cell expression of PD-1 is minimal to nonexistent, particularly when compared with either TIGIT or to PD-1 expression by T cells.

\section{Discussion}

In this study, we attempted to thoroughly evaluate the expression of the immune checkpoint marker PD-1 on NK cells under diverse conditions in human, mouse, and even canine models using multiple readouts. We consistently observed minimal expression of PD-1 on NK cells despite showing robust NK cell activation, proliferation, and function across these multiple species and models (in which T cell upregulation of PD-1 consistently occurred). As the majority of studies solely used flow cytometry to determine PD-1 expression, we used different PD- 1 hybridomas and validated our results using both qRT-PCR and RNA-Seq techniques - in addition to analyzing NK cells from $\mathrm{T}$ and $\mathrm{B}$ cell-deficient $\mathrm{Rag}^{-{ }^{--}}$mice and PD-1 reporter mice. The reporter mice provided a unique model for evaluating PD-1 expression, including cell surface and intracellular, without dependence on monoclonal antibodies, and importantly, we confirmed our results from the other models. Overall, our data suggest that incremental changes in flow cytometric expression (as observed by us and others; ref. 12, 24) may be subject to multiple confounding factors. Tumor inflammatory cell presence and tissue specimen processing have been shown to interfere with lymphocyte marker expression, notably on CD3 on T cells $(61,62)$, which may result in false-positive results, especially when small differences are detected. The effects of limited cellular events in flow cytometry also raise concerns with regard to specificity of binding, especially given the limited numbers of NK cells observed in the majority of tumor samples from both mouse and human. The majority of mouse preclinical studies employ tumor cell lines injected subcutaneously, and in these models, NK cell trafficking and homing are minimal, especially when compared with $\mathrm{T}$ cells.

Although the impact of PD-1 inhibition on T cell exhaustion is well characterized, less is known regarding exhaustion in NK cell biology. There is substantial evidence that immunoregulatory molecules, such as TIGIT, which we have observed consistently upregulated, as well as other molecules, such as TIM-3 $(21,63)$, LAG-3 (34), and PD-L1 (32, 64), appear more robustly and consistently expressed on NK cells. NK cells and T cells also differ in their regulation in how they signal. In contrast to that of $\mathrm{T}$ cells, the functional status of NK cells is regulated by the cumulative effect of multiple activating and inhibitory signals, rather than direct competition between antigen-triggered costimulatory and coinhibitory receptors (such as CD28 and PD-1) (65). These critical differences between NK cells and T cells, combined with the marked expression of these other inhibitory molecules, raise the question of what physiologic role PD-1 would play on NK cells $(66,67)$. These questions are further confounded by studies showing PD-1 expression exclusively on activated NK cells (23), while others identify PD-1 expression on NK cells with a dysfunctional phenotype $(12,29)$.

There is also the context in which PD- 1 has been detected, given either the highly inflammatory nature of the model (i.e., MCMV infection) or the TME $(12,24)$, and this has led to variability in 
A

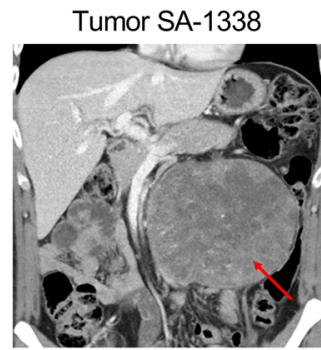

B

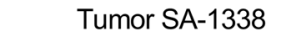

Round Cell Sarcoma

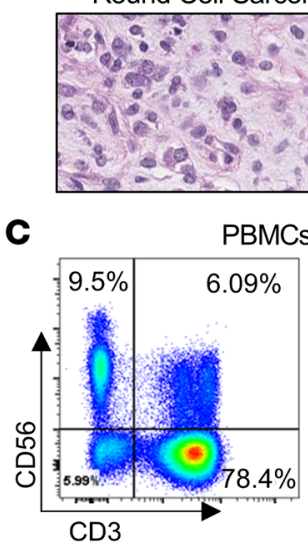

C
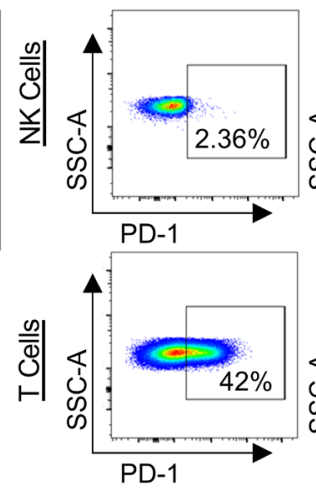

Tumor SA-1206

Pleomorphic Sarcoma

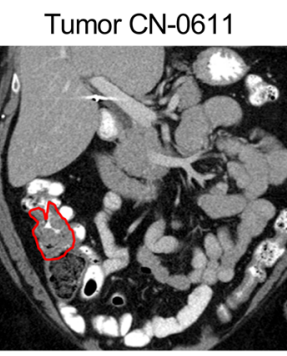

Tumor $\mathrm{CN}-0611$

Colon Adenocarcinoma
Tumor $\mathrm{CN}-0611$

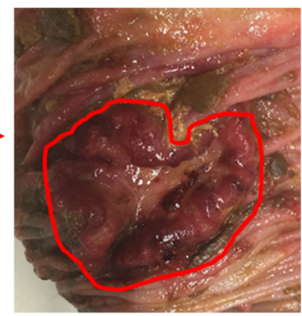


Figure 7. Tumor-infiltrating NK cells from human tumors show increased TIGIT and minimal PD-1 expression. TILs were analyzed from human tumor specimens for expression of PD-1 and TIGIT and compared with matched circulating PBMCs. (A) Preoperative CT, PET/CT, and intraoperative surgical specimens are shown along with (B) representative $\mathrm{H} \& \mathrm{E}$ staining. Representative flow cytometry shows NK and T cell populations with PD-1 and TIGIT expression on NK and T cells from (C) peripheral blood and (D) tumor from a round cell sarcoma patient. (C and D) PD-1 expression on peripheral and intratumoral NK cells is minimal compared with expression on T cells, while TIGIT is expressed on both NK and T cells. Representative staining from (E) peripheral blood and (F) tumor from a patient with colon cancer shows a higher proportion of NK cells compared with sarcomas. (C) No upregulation of PD-1 was detected on infiltrating NK cells compared with peripheral NK cells, while T cell PD-1 expression is significantly upregulated. (H) TIGIT is highly expressed on NK and T cells in both the periphery and tumor. (I) PD-1 expression was minimal on NK cells in all tumors analyzed, while PD-1 expression was significantly higher on intratumoral T cells. (J) TIGIT is expressed on both NK and T cells in all tumors analyzed. Data are shown as mean \pm SD for $n=7$ matched PBMCs and tumors. ${ }^{*} P<0.05 ;{ }^{* *} P<0.001$, paired Student's $t$ test.

reported PD-1 expression across tissue type and models. Another caveat revolves around attempting to ascribe effects solely to NK cells. In vivo NK depletion studies using aASGM1 are problematic, given the lack of specificity. As we and others have shown, depletion of ASGM1 ${ }^{+}$cells markedly affects the effector function of activated T cells (among others) (40-42), highlighting important differences between resting/activated immune states. Although NK1.1 depletion appears more selective for NK cell depletion, it also depletes NKT cells and, as we observed here, appears to have nonspecific effects on activated T cells as well. Therefore, it is difficult to categorize any of these depletion strategies as truly "NK specific," and careful controls for T cell effects or use of T celldeficient mice should be considered.

Finally, an important limitation of studies showing expression of PD-1 by NK cells is the almost exclusive reliance on flow cytometry. Although flow cytometry clearly provides high-dimensional and highly granular data, determination of whether cells "positively" or "negatively" bind fluorescently labeled antibodies to molecules of interest remains based on manual gating by visual inspection. Despite the sophisticated nature of data acquisition by flow cytometry, gating strategies for data interpretation remain prone to potential bias and/or subjectivity. In fact, the variable nature of manual gating strategies has been shown to lead to disparate results even when experienced researchers have been provided identical data sets (68). In this study, a substantial portion of interlaboratory variability in flow cytometry data analysis could be eliminated by centralization of data interpretation in one or a handful of selected laboratories along with the use of a dynamic gating strategy based on computer-generated clustering algorithms (68). An additional pitfall of flow cytometry is the potential for changes in the molecular or metabolic condition of cells (such as highly inflammatory environment of acute viral infection or when tumor necrosis is present) to alter the binding properties of either the antibodies or the epitopes of interest, leading to variable results or possible false-positive staining. This technical limitation of flow cytometry was recently detailed, showing that dying immune and tumor cells unmask and express a nuclear antigen that cross-reacts with mouse anti-PD-1 mono- clonal antibodies and thereby provides a source of false-positive PD-1 expression (69). This is further complicated by the cellular phenomenon of trogocytosis, in which extracted proteins can be expressed on a cell's surface, which has been identified in NK cells, particularly when activated $(70,71)$. In cases where the data obtained are difficult to reconcile, especially in the case of PD-1 expression by NK cells, these technical caveats can be noteworthy, emphasizing the importance of multiple experimental techniques. This is also important considering the expression of the Fc receptor CD16 by NK cells, allowing for even greater chances for false-positive antibody binding.

Although we cannot completely eliminate the potential for NK cells to express PD- 1 on an extremely small fraction of NK cells under highly selected situations, our data from both mouse and human tumors show that the physiological impact and therefore efficacy of such an approach is likely minimal. It is difficult to envision a situation where a single digit fraction of $\mathrm{PD}-1^{+} \mathrm{NK}$ cells of an even smaller subpopulation of intratumoral NK cells are mediating biologically significant antitumor effects. As a result, given our extensive analysis of multispecies NK cells in diverse viral, tumor, and cytokine-activated models showing minimal expression of PD-1 on NK cells, our data do not support direct effects of anti-PD-1 therapy on augmenting NK responses. It is conceivable that PD-1 and PD-L1 inhibition may exert indirect effects on NK effector function, leading to heightened NK responses independent of direct expression of PD- 1 by NK cells. As PD-L1 is frequently expressed on tumor cells, blocking anti-PD-L1 antibodies (as well as other monoclonal antibodies) may activate NK cells via antibody-dependent cellular cytotoxicity (ADCC) rather than intrinsic signaling via the PD-1 pathway. It is also possible that the effects of PD-1 or PD-L1 inhibition on NK cells could be mediated through PD-L1 signaling on NK cells or through augmenting paracrine or endocrine signaling by $\mathrm{T}$ cells. Certainly, the inhibitory interaction of PD-L1+ liver-resident NK (LrNK) cells against PD- $1^{+}$T cells in the response to viral hepatitis showing that LrNK cells inhibit $\mathrm{T}$ cells suggests that PD-L1 expression by NK cells may be responsible for many effects of checkpoint inhibition (64). Similarly, other investigators identified a PD-1-independent mechanism of antitumor efficacy via the activation of PD-L1 ${ }^{+}$NK cells using an anti-PD-L1 antibody, providing another potential explanation for why some patients who lack PD-L1 expression on tumor cells may still respond to immune checkpoint inhibition (32). Finally, while studies of PD-1 expression on NK cells are mixed, there are other studies that have observed robust expression of checkpoint markers, such as TIGIT, TIM-3, and LAG-3, as critical regulators of NK function $(21,31,34)$.

Given the intense interest in clinical strategies to augment NK function and the use of CAR-NK cells, we feel that our data are both timely and important. In contrast to seminal studies on $\mathrm{T}$ cell exhaustion that have focused on viral models and human patients with chronic viral infections, studies investigating NK cell exhaustion have focused primarily on identifying dysfunctional intratumoral NK cells and applying strategies to reverse NK exhaustion to augment antitumor effects. However, the extent of NK infiltration in solid tumors is generally low (72), and the clinical relevance of intratumoral NK cells remains incompletely characterized, further confounding interpretation of smaller subpopulations expressing certain molecules. 
A

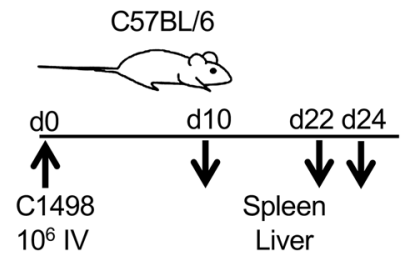

B

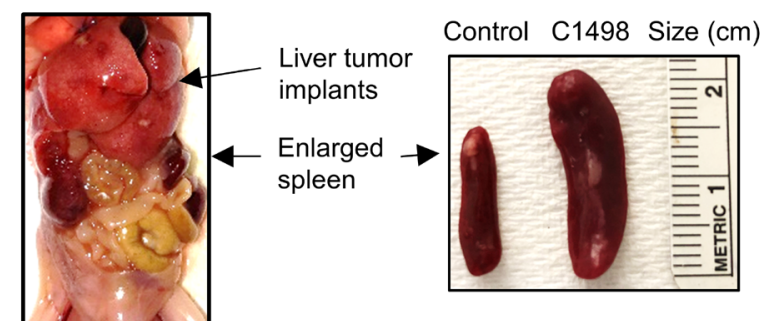

C

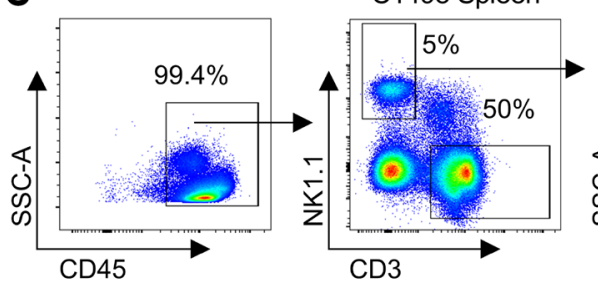

E

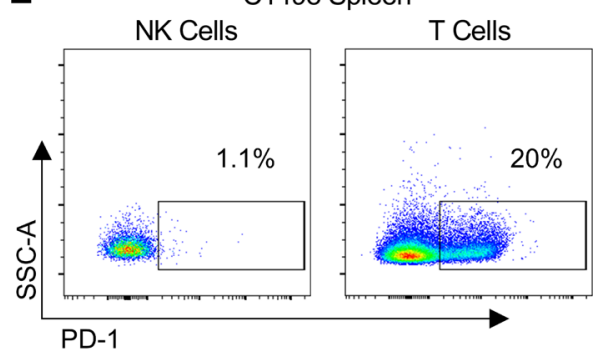

H
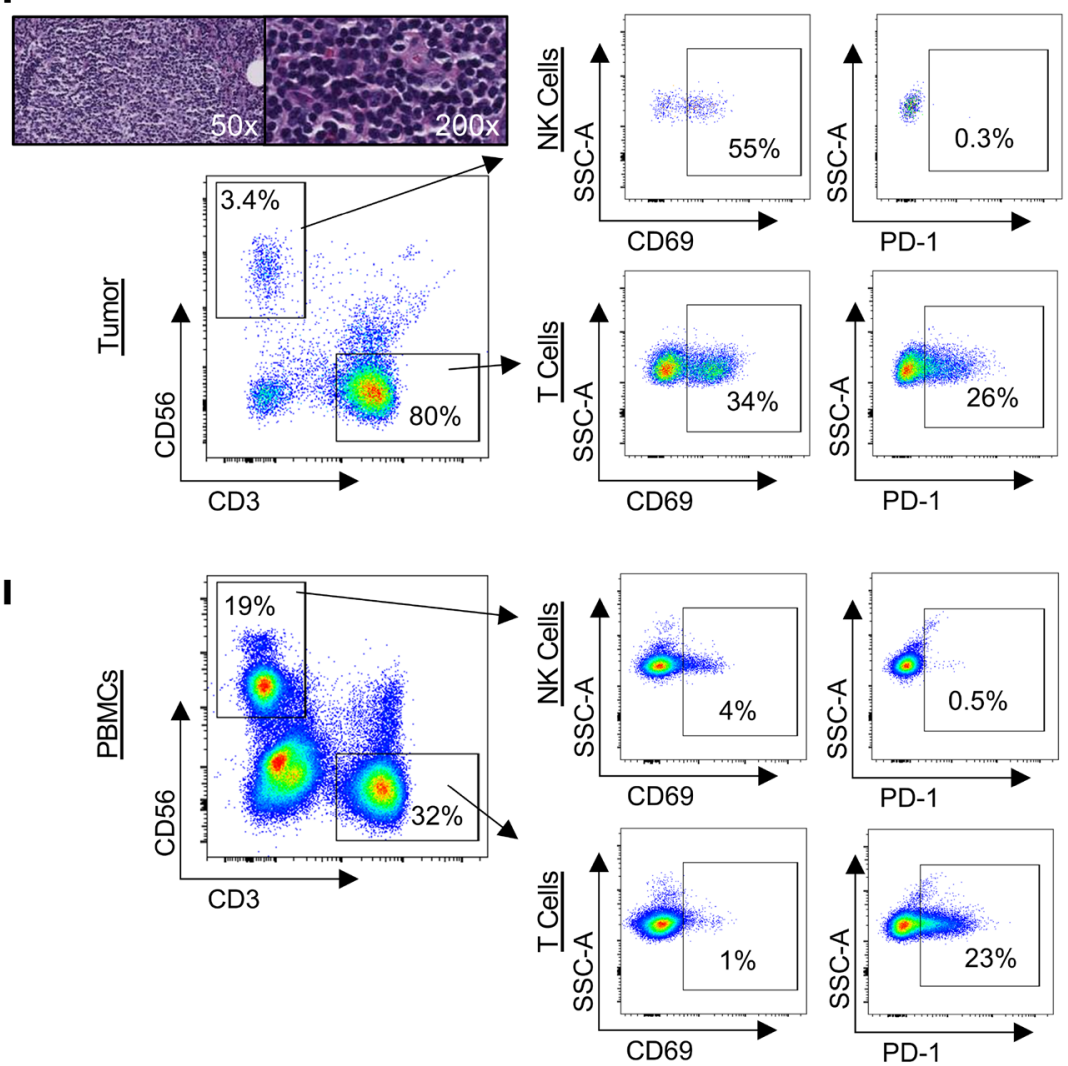

D

$\mathbf{F}$
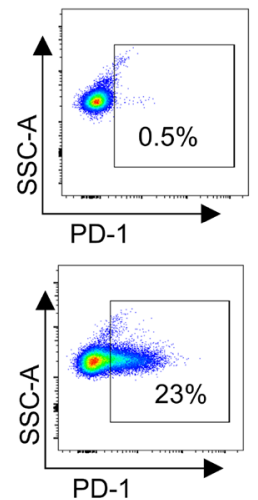
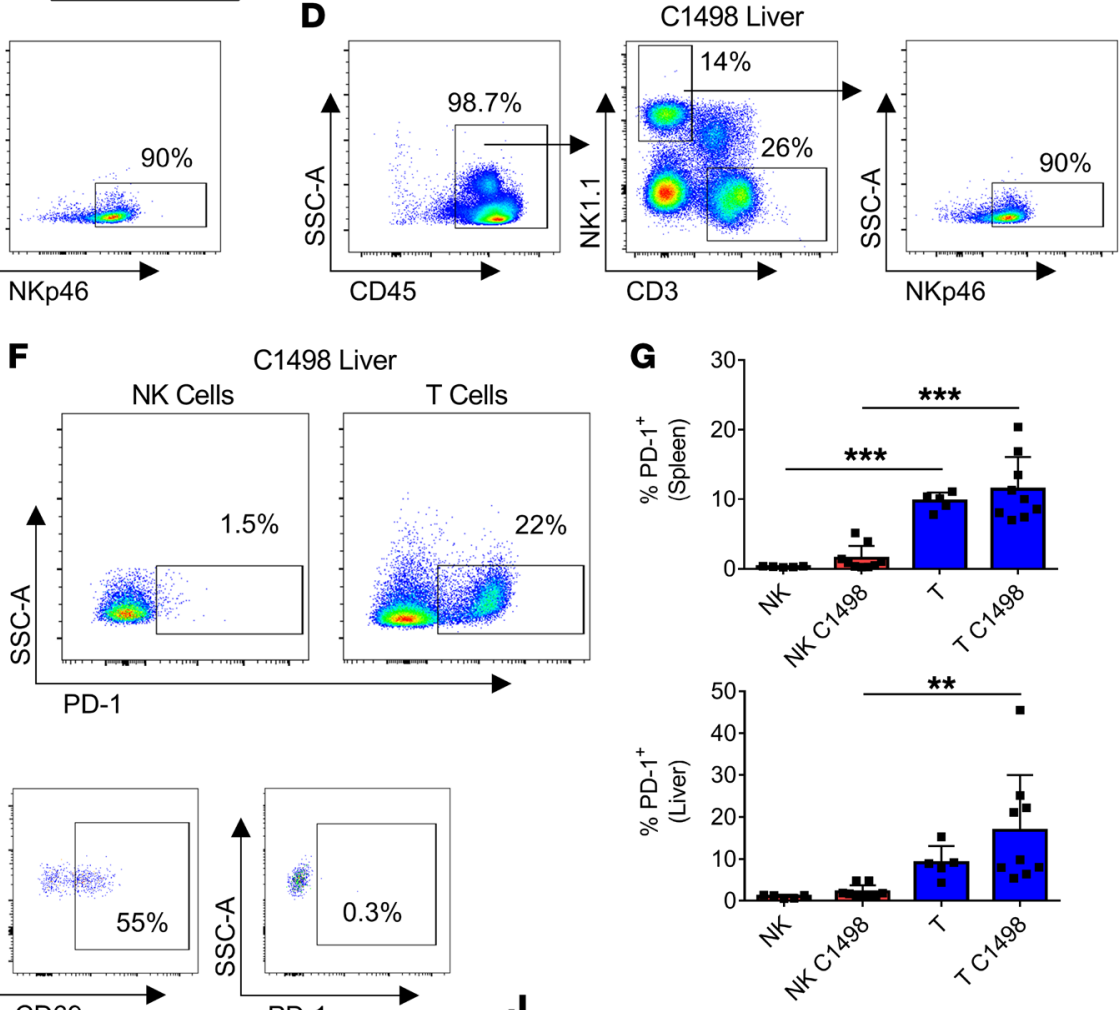

J

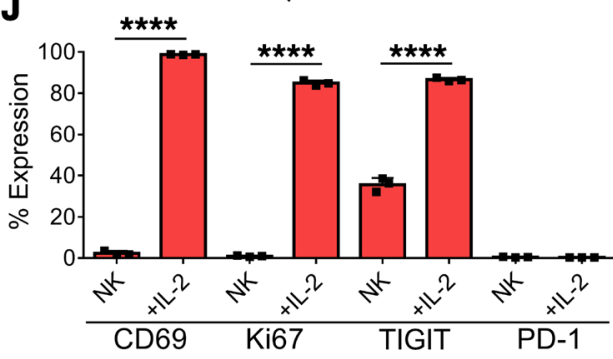

$\mathbf{K}$

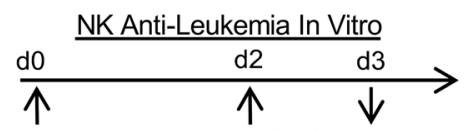

Human NK cells \pm K562 (1:1) Flow cytometry $\pm 1000 \mathrm{IU} / \mathrm{mL} \mathrm{IL}-2 \quad$ analysis 
Figure 8. NK cells activated by hematologic malignancies exhibit minimal PD-1 expression. (A) Schematic depicts metastatic AML mouse model. (B) Gross pathology shows diffuse tumor implants in the liver and splenomegaly compared with control mice (PBS injection). Days 22-24 gating for analysis of NK (CD3-NK1.1+NKp46+) and T (CD3+NK1.1 ) cells from the (C) spleen and (D) liver. NK cells isolated from the (E) spleen and (F) liver exhibit minimal PD-1 expression compared with T cells. (G) Summary data show $\mathrm{PD}-1$ expression on NK and T cells from all mice analyzed. Tumor-infiltrating NK and T cells were analyzed from a patient undergoing a lymph node biopsy for recurrent follicular lymphoma. (H) H\&E staining shows follicular lymphoma architecture. Parent gating from the tumor shows NK and T cell populations and increased expression of CD69 on the infiltrating NK and T cells. PD-1 expression is minimal on the infiltrating NK cells. (I) NK and T cell populations from peripheral blood exhibit minimal CD69 expression and minimal PD-1 expression on peripheral NK cells. (J) PBMCs isolated from the patient were cultured for 72 hours in either media alone (NK) or $1000 \mathrm{IU} /$ $\mathrm{mL}$ rhIL-2 (+IL-2). Cytokine-stimulated NK cells upregulated expression of CD69, Ki67 and TICIT, while PD-1 expression remained minimal. (K) Isolated NK cells from a healthy human donor were cultured for 48 hours in media alone or $1000 \mathrm{IU} / \mathrm{mL}$ rhIL-2 and then incubated with $\mathrm{K} 562$ target cells. Culture with target cells led to robust CD107a expression, but PD-1 expression remained minimal. (G) Data are shown as mean \pm SD for $n=5-9$ mice/ group. (J) Single patient in triplicate in vitro. (K) $n=3$ /group, representative of 2 independent experiments using separate healthy human donors. ${ }^{* * P}$ $<0.01 ;{ }^{* * *} P<0.001 ;{ }^{* * *} P<0.0001$, 1-way ANOVA with multiple group comparisons (G) or unpaired Student's $t$ test (J).

Overall, our results highlight the growing body of literature showing the absence of significant PD-1 expression on mouse, canine, and human NK cells under multiple heterogeneous conditions where NK cells are highly activated $(21,69,73)$. A greater understanding of NK cell biology as well as the expression and functional implications of inhibitory markers is critical to advances in the clinical application of NK immunotherapy.

\section{Methods}

Mice. Female C57BL/6J and BALB/cJ mice were purchased from The

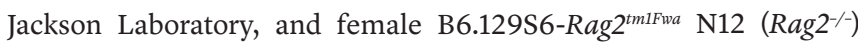
mice were purchased from Taconic Farms. Unless otherwise noted, mice were 2 to 4 months old at time of experimentation. DIO and control mice were generated as previously described (36) and used when they were approximately 9 months old.

Mouse in vitro studies. Adherent lymphokine-activated killer cells from mouse splenocytes were generated using recombinant human IL-2 (teceleukin, National Cancer Institute [NCI]) at indicated concentrations. Half-volume media changes were performed every 2 to 3 days.

In vivo mouse tumor studies. Mouse tumor cell lines were thawed and passaged in complete RPMI media supplemented with $10 \%$ $\mathrm{Nu}$-Serum (Corning Life Sciences) for 3 passages before usage. The $\mathrm{BALB} / \mathrm{c}$ TdTomato reporter mice were injected with $5 \times 10^{5} \mathrm{CT} 26$ cells (ATCC, CRL-2638) in $100 \mu \mathrm{L}$ PBS. Mice were euthanized and tissue collected at indicated time points or earlier if prespecified criteria were met. Additional details are provided in Supplemental Methods.

Isolation of murine TILs. After euthanasia, tumors were removed and mechanically dissociated in PBS. Tissue was then strained through a $70 \mu \mathrm{m}$ filter, and cells were treated with RBC lysis buffer for 5 minutes (BioLegend, catalog 420301). For the BALB/c TdTomato reporter mice, mice were euthanized and tumors removed and manually digested, then enzymatically digested with Liberase (Roche), DNAse I (Sigma-Aldrich), hyaluronidase (Sigma-Aldrich), and collagenase XI
(Sigma-Aldrich). Single-cell suspensions were enriched for lymphocytes by isolating cells at the interface of $44 \%$ Percoll (Sigma-Aldrich) in media and Lympholyte-M (Cedarlane) gradient.

MCMV infection. MCMV Smith strain was obtained from ATCC and maintained by repeated salivary gland passage in female BALB/c mice. Mice were infected with MCMV via intraperitoneal injection of virus in $200 \mu \mathrm{L}$ RPMI 1640 at the doses specified. Control mice were injected with $200 \mu \mathrm{L}$ RPMI 1640 only. Liver tissue samples were snap-frozen for MCMV viral titer quantification via real-time PCR. IE-1 copy numbers were normalized to DNA quantity and tissue weight (25 $\mathrm{mg}$ for liver).

In vivo NK cell depletion. We performed in vivo depletions by intraperitoneal injection of anti-ASGM1 (Wako Chemicals, catalog 98610001) or anti-NK1.1 (clone PK136, National Cell Culture Center) or by sham injection of PBS. Anti-ASGM1 was prepared with $10 \mu \mathrm{L}$ stock in $200 \mu \mathrm{L}$ PBS per mouse. Anti-NK1.1 was prepared with $300 \mu \mathrm{g}$ stock in $200 \mu \mathrm{L}$ PBS. Injections were performed twice -2 days before $1 \times$ $10^{4}$ PFU MCMV infection and 5 days following infection.

Processing of mouse tissue. Following euthanasia, organs were collected and processed. Liver hematopoietic cells were enriched by density centrifugation with $32 \%$ Percoll in wash buffer (3\% Nu-Serum in PBS). Cells were treated with RBC lysis buffer for 5 minutes, washed, then resuspended in PBS for analysis.

NK cells in vitro. Human leukocyte reduction system chambers were obtained from healthy, unidentified volunteers (BloodSource, Sacramento, California, USA). PBMCs were isolated using a density gradient (Lymphocyte Separation Medium, Corning Life Sciences), followed by RBC lysis. Human NK cells were isolated from PBMCs using the RosetteSep Human NK Isolation Kit according to the manufacturer's specifications (Stem Cell Technologies). Following isolation, human NK cells were immediately used or stored in liquid nitrogen. Human PBMCs and NK cells were cultured in incubators at $37^{\circ} \mathrm{C}$ at $5 \%$ $\mathrm{CO}_{2}$ in complete RPMI media supplemented with cytokines. Recombinant human IL-2 and IL-15 were obtained from the NCI Biological Resources Branch. ConA was obtained from Sigma-Aldrich (catalog C5275). Human NK cells were grown in complete RPMI media and cocultured with irradiated K562.Clone 9-mIL21-41BBL cells (courtesy of Dean Lee, Nationwide Children's Hospital, Columbus, Ohio, USA) and $100 \mathrm{IU} / \mathrm{mL}$ recombinant human IL-2 (NCI) (74). K562. Clone 9-mIL21-41BBL cells were treated with 100 Gy using a Mark I $\mathrm{Cs}^{137}$ irradiator (JL Shepherd \& Associates). NK92 cells were obtained from ATCC and cultured in complete RPMI media supplemented with $500 \mathrm{IU} / \mathrm{mL}$ recombinant human IL-2. Cells were passaged every 3 to 5 days and used within the first 10 passages. Canine NK cells were obtained from healthy farm beagles and isolated and expanded as described previously (51). The human erythroleukemia cell line K562 was obtained from the NCI and cultured in RPMI complete media. For studies of CD107a, anti-CD107a antibody was added in vitro on the day of analysis. After 1 hour, brefeldin A (Golgi Plug, BD Biosciences) and monensin (Golgi Stop, BD Biosciences) were added per the manufacturer's recommendations. After an additional 3 to 5 hours, cells were collected and prepared for flow cytometry.

Human clinical tumor and peripheral blood samples. Human study participants were both male and female donors undergoing scheduled surgical resection of various cancers through the UCD Comprehensive Cancer Center. Additional details are provided in Supplemental Methods. 
RNA isolation, reverse transcription, and qRT-PCR. RNA was isolated using the RNeasy Mini Kit (QIAGEN) following the manufacturer's protocol with optimal on-column DNAse treatment to remove genomic DNA. RNA concentrations were quantified using a Qubit 4 fluorometer, and cDNA was synthesized using Iscript Reverse Transcription Supermix (Bio-Rad). mRNA expression was detected using qRT-PCR via specified primers for human, mouse, and canine expression (Supplemental Table 1) on the Bio-Rad CFX384 system.

RNA-Seq. Resting and rhIL-2-stimulated NK cells were obtained from the spleens of WT C57BL/6 mice (75). Stimulated NK cells were generated by isolating splenocytes from C57BL/6 mice $(n=30)$, pooling, then culturing in $1000 \mathrm{IU} / \mathrm{mL}$ rhIL-2 for 7 days. Following collection, single-cell suspensions were generated and $\mathrm{Fc}$ receptors blocked (anti-CD16/32, clone 93, BioLegend) and stained for NK isolation. $\mathrm{CD}^{-} \mathrm{CD} 122^{+}$cells were isolated using a FACS Aria machine (BD Biosciences). Sorted cells were then processed for RNA extraction using the QIAGEN RNeasy Kit. Intratumoral NK and T cells were obtained from subcutaneous CT26 tumors implanted in WT BALB/c mice $(n=$ 4-8/group), which were excised and pooled. Following collection, single-cell suspensions were generated, Fc receptors blocked, and cells stained. Cells were sorted using a FACS Aria (BD Biosciences) for intratumoral $\mathrm{NK}\left(\mathrm{CD} 45^{+} \mathrm{CD} 3^{-} \mathrm{NKp} 46^{+}\right)$and $\mathrm{T}\left(\mathrm{CD} 45^{+} \mathrm{CD}^{+} \mathrm{NKp} 46^{-}\right)$ cells. Cells were processed for RNA isolation using the Total RNA Purification Plus Micro Kit (Norgen Biotek). RNA-Seq libraries were prepared by the UCD Bioinformatics Core.

Serum corticosterone. Serum was isolated by centrifugation of peripheral blood obtained via tail vein bleed. Measurement of serum corticosterone was performed by the UCD Mouse Biology Program Metabolomics Core using the ImmuChem Double Antibody Corticosterone $\mathrm{I}^{125}$ RIA Kit (MP Biomedicals) and calibrated to a standard curve.

Antibody staining and flow cytometry. Human cells were prepared in a single-cell suspension. Cells were washed with PBS, incubated with Human TruStain Fc Receptor Blocking Solution (BioLegend, 422302), then stained with the following fluorochrome-conjugated monoclonal antibodies: CD3-FITC (clone HIT3a, BioLegend), CD56-PE (clone HCD56, BioLegend), PD-1-APC (clone EH12.2H7, BioLegend), TIGIT-BV421 (clone A15153G, BioLegend), CD69BV711 (clone FN50, BioLegend), CD107a-BV605 (clone H4A3, BioLegend), Ki-67-PE/Cy7 (clone 20Raj1, eBioscience), CD45-BV510 (clone HI30, BioLegend), and granzyme B-APC (clone GB12, Invitrogen). Live/dead staining was performed using Fixable Viability Dye 780 (eBioscience).

Mouse cells were prepared in a single-cell suspension, washed with PBS, incubated with Fc block (anti-CD16/32, clone 93, BioLegend), then stained with the following fluorochrome-conjugated antibodies: CD45-PacBlue (clone 30-F11, BioLegend), CD45-FITC (clone 30-F11, Thermo Fisher Scientific), CD3-BV785 (clone 17A2, BioLegend), CD3-Pacific Blue (clone 17A2, Thermo Fisher Scientific), NKp46-PerCP-Cy5.5 (clone 29A1.4, BioLegend), CD122-APC (clone TM- $\beta 1$, BioLegend), NKp46-APC (clone 29A1.4, BioLegend), PD-1-PE/Cy7 (clone RMP1-30, BioLegend), TIGIT-APC (clone 1G9, BioLegend), CD49b-PerCP-Cy5.5 (clone HMa2, BioLegend), NK1.1PE-TR (clone PK136, Thermo Fisher Scientific), CD69-FITC (clone H1.2F3, BD Biosciences), NKp46-PerCP-Cy5.5 (clone 29A1.4, BD Biosciences), Thy1.2-APC (clone 53-2.1, eBioscience), and granzyme B-FITC (clone GB11, BioLegend). AF594 goat anti-rabbit secondary antibody was used for ASGM1 detection (Jackson ImmunoResearch, catalog 111-586-003). The following additional antibodies against murine PD-1 were used: PD-1-BV711 (clone 29F.1A12, BioLegend), PD-1-FITC (clone J43, eBioscience), and PD-1-PE (clone J43, eBioscience). Live/dead staining was performed using Aqua Viability Dye (Thermo Fisher Scientific). All intracellular staining was completed using the BD Biosciences Fixation/Permeabilization Solution Kit. All human and mouse cells were analyzed using a BD LSRFortessa flow cytometer (BD) and FlowJo Software (BD).

RNA-Seq data were deposited in the NCBI's Gene Expression Omnibus database (GEO GSE145837).

Statistics. Prism software (GraphPad Software Inc.) was used for graph generation and statistical analysis. Data are expressed as mean $\pm \mathrm{SD}$. Where appropriate, normality of distribution was confirmed using the Shapiro-Wilk normality test. For analysis of 3 or more groups, 1-way ANOVA test was performed with Tukey's or Dunnett's post test as appropriate. Differences between 2 normally distributed groups were analyzed using the paired or unpaired Student's $t$ test as appropriate. $P \leq 0.05$ was considered statistically significant.

Study approval. Mice were housed in AAALAC-accredited animal facilities at UCD under specific pathogen-free conditions. All animal experiments were reviewed and approved by the Animal Care and Use Committee at UCD, University of North Carolina at Chapel Hill, or the $\mathrm{NCI}$, in accordance with the guidelines of the NIH. For human tumor studies, collection of matched whole blood and tumor specimens was approved by the IRB at UCD (protocol 218204-9).

\section{Author contributions}

SJJ, SCV, JSS, WJM, and RJC designed the study. SJJ, CD, EGA, SCV, IRS, and SKA conducted the experiments and collected the data. IRS, KMS, and JVD provided technical assistance. SJJ, SCV, JSS, WJM, and RJC analyzed the data. SJJ, WJM, and RJC wrote the manuscript. All authors, including LTK, DLL, MAD, BRB, and AMM, provided critical reviews of the manuscript.

\section{Acknowledgments}

This work was supported in part by NIH/NCI grant R01 HL140921 (to WJM); P50 CA058223 (to JSS); R01 HL56067, R37 AU34494, and 2P01 CA065493 (to BRB); and U01 CA224166-01 and the Robert Lambert Family Fund (to RJC). This work was also supported in part by funds from the UCD Comprehensive Cancer Center and the UCD Flow Cytometry Shared Resource Laboratory, with funding from NCI P30 CA093373 (Cancer Center), S10 OD018223 (Astrios Cell Sorter), and S10 RR 026825 (Fortessa Cytometer) grants, with technical assistance from Bridget McLaughlin and Jonathan Van Dyke. Specimens were provided by the UCD Pathology Biorepository, which is jointly funded by the UCD Comprehensive Cancer Support Grant (CCSG) awarded by the NCI (P30 CA093373) and the UCD Department of Pathology and Laboratory Medicine. We give special thanks to Wissam J. Halabi (UCD Division of Colorectal Surgery), Sepideh Gholami (UCD Division of Surgical Oncology), Andrew Birkeland (UCD Department of Otolaryngology), and the past and current members of the Murphy lab for helpful discussions.

Address correspondence to: William J. Murphy, 2921 Stockton Boulevard, Room 1614, Sacramento, California 95817, USA. Phone: 916.703.9397; Email: wmjmurphy@ucdavis.edu. 
1. Sharma P, Allison JP. The future of immune checkpoint therapy. Science. 2015;348(6230):56-61.

2. Wherry EJ. T cell exhaustion. Nat Immunol. 2011;12(6):492-499.

3. Wherry EJ, Kurachi M. Molecular and cellular insights into T cell exhaustion. Nat Rev Immunol. 2015;15(8):486-499.

4. Hashimoto M, et al. CD8 T cell exhaustion in chronic infection and cancer: opportunities for interventions. Аnпи Rev Med. 2018;69:301-318.

5. Agata Y, et al. Expression of the PD-1 antigen on the surface of stimulated mouse $T$ and $B$ lymphocytes. Int Immunol. 1996;8(5):765-772.

6. Thibult ML, et al. PD-1 is a novel regulator of human B-cell activation. Int Immunol. 2013;25(2):129-137.

7. Yu Y, et al. Single-cell RNA-seq identifies a PD-1 ${ }^{\text {hi }}$ ILC progenitor and defines its development pathway. Nature. 2016;539(7627):102-106.

8. Liu Y, et al. Regulation of arginase I activity and expression by both PD- 1 and CTLA- 4 on the myeloid-derived suppressor cells. Cancer Immunol Immunother. 2009;58(5):687-697.

9. Gordon SR, et al. PD-1 expression by tumour-associated macrophages inhibits phagocytosis and tumour immunity. Nature. 2017;545(7655):495-499.

10. Cho HY, Lee SW, Seo SK, Choi IW, Choi I, Lee SW. Interferon-sensitive response element (ISRE) is mainly responsible for IFN-alphainduced upregulation of programmed death-1 (PD-1) in macrophages. Biochim Biophys Acta. 2008;1779(12):811-819.

11. Davis ZB, Vallera DA, Miller JS, Felices M. Natural killer cells unleashed: Checkpoint receptor blockade and BiKE/TriKE utilization in NK-mediated anti-tumor immunotherapy. Semin Immunol. 2017;31:64-75.

12. Hsu J, et al. Contribution of NK cells to immunotherapy mediated by PD-1/PD-L1 blockade. JClin Invest. 2018;128(10):4654-4668.

13. Bachanova V, Miller JS. NK cells in therapy of cancer. Crit Rev Oncog. 2014;19(1-2):133-141.

14. Knorr DA, Bachanova V, Verneris MR, Miller JS. Clinical utility of natural killer cells in cancer therapy and transplantation. Semin Immunol. 2014;26(2):161-172.

15. Ruggeri $\mathrm{L}$, et al. Effectiveness of donor natural killer cell alloreactivity in mismatched hematopoietic transplants. Science. 2002;295(5562):2097-2100.

16. Miller JS, et al. Successful adoptive transfer and in vivo expansion of human haploidentical NK cells in patients with cancer. Blood 2005;105(8):3051-3057.

17. Bachanova V, et al. Haploidentical natural killer cells induce remissions in non-Hodgkin lymphoma patients with low levels of immune-suppressor cells. Cancer Immunol Immunother. 2018;67(3):483-494.

18. Locatelli F, et al. Cellular and molecular basis of haploidentical hematopoietic stem cell transplantation in the successful treatment of highrisk leukemias: role of alloreactive NK cells. Front Immunol. 2013;4:15

19. Locatelli F, Pende D, Maccario R, Mingari MC, Moretta A, Moretta L. Haploidentical hemopoietic stem cell transplantation for the treatment of high-risk leukemias: how NK cells make the difference. Clin Immunol. 2009;133(2):171-178.

20. Bi J, Tian Z. NK cell exhaustion. Front Immunol. 2017;8:760.

21. da Silva IP, et al. Reversal of NK-cell exhaustion in advanced melanoma by Tim-3 blockade. Cancer Immunol Res. 2014;2(5):410-422.

22. Alvarez M, et al. Regulation of murine NK cell exhaustion through the activation of the DNA damage repair pathway. JCI Insight. 2019;5:127729.

23. Lieberman NAP, et al. An uncoupling of canonical phenotypic markers and functional potency of ex vivo-expanded natural killer cells. Front Immunol. 2018;9:150.

24. Quatrini L, et al. Endogenous glucocorticoids control host resistance to viral infection through the tissue-specific regulation of PD-1 expression on NK cells. Nat Immunol. 2018;19(9):954-962.

25. A pilot study of NK cell combined with PD-1 antibody as second line therapy for advanced driver mutation negative non-small cell lung cancer. http://clinicaltrials.gov. NCT03958097. Accessed March 23, 2020.

26. A study of PD1 combined with DC-NK cell in the treatment of solid tumors. http://clinicaltrials.gov. NCT03815084. Accessed March 23, 2020.

27. Allogeneic NK cell ("SMT-NK”) in combination with pembrolizumab in advanced biliary tract cancer. http://clinicaltrials.gov. NCT03937895. Accessed March 23, 2020.

28. Li Q, et al. Abstract A014: Phase I clinical trial with PD-1/MUC1 CAR-pNK92 immunotherapy. Cancer Immunol Res. 2019; 7(2 Suppl):A014.

29. Beldi-Ferchiou A, et al. PD-1 mediates functional exhaustion of activated NK cells in patients with Kaposi sarcoma. Oncotarget. 2016;7(45):72961-72977.

30. MacFarlane AW, et al. PD-1 expression on peripheral blood cells increases with stage in renal cell carcinoma patients and is rapidly reduced after surgical tumor resection. Cancer Immunol Res. 2014;2(4):320-331.

31. Zhang $\mathrm{Q}$, et al. Blockade of the checkpoint receptor TIGIT prevents NK cell exhaustion and elicits potent anti-tumor immunity. Nat Immunol. 2018;19(7):723-732.

32. Dong W, et al. The mechanism of anti-PD-L1 antibody efficacy against PD-L1-negative tumors identifies NK cells expressing PD-L1 as a cytolytic effector. Cancer Discov. 2019;9(10):1422-1437.

33. Datar I, et al. Expression analysis and significance of PD-1, LAG-3, and TIM-3 in human nonsmall cell lung cancer using spatially resolved and multiparametric single-cell analysis. Clin Cancer Res. 2019;25(15):4663-4673.

34. Merino A, et al. Chronic stimulation drives human NK cell dysfunction and epigenetic reprograming. J Clin Invest. 2019;130:3770-3785.

35. Gunji Y, Vujanovic NL, Hiserodt JC, Herberman RB, Gorelik E. Generation and characterization of purified adherent lymphokine-activated killer cells in mice. J Immunol. 1989;142(5):1748-1754.

36. Wang Z, et al. Paradoxical effects of obesity on $\mathrm{T}$ cell function during tumor progression and PD-1 checkpoint blockade. Nat Med.2019;25(1):141-151.

37. Arase H, Lanier LL. Virus-driven evolution of natural killer cell receptors. Microbes Infect. 2002;4(15):1505-1512.
38. Arase H, Mocarski ES, Campbell AE, Hill AB, Lanier LL. Direct recognition of cytomegalovirus by activating and inhibitory NK cell receptors. Science. 2002;296(5571):1323-1326.

39. Cerwenka A, Lanier LL. Natural killer cell memory in infection, inflammation and cancer. Nat Rev Immunol. 2016;16(2):112-123.

40. Nishikado H, Mukai K, Kawano Y, Minegishi Y, Karasuyama H. NK cell-depleting anti-asialo GM1 antibody exhibits a lethal off-target effect on basophils in vivo. JImmunol. 2011;186(10):5766-5771.

41. Charley MR, Mikhael A, Hackett J, Kumar V, Bennett M. Mechanism of anti-asialo GM1 prevention of graft-vs-host disease: identification of allo-antigen activated T cells. J Invest Dermatol. 1988;91(3):202-206.

42. Wiltrout RH, et al. Reactivity of anti-asialo GM1 serum with tumoricidal and non-tumoricidal mouse macrophages. JLeukoc Biol. 1985;37(5):597-614.

43. Slifka MK, Pagarigan RR, Whitton JL. NK markers are expressed on a high percentage of virus-specific CD8+ and CD4+ T cells. JImmunol. 2000;164(4):2009-2015.

44. Moore ML, Chi MH, Goleniewska K, Durbin JE, Peebles RS. Differential regulation of GM1 and asialo-GM1 expression by T cells and natural killer (NK) cells in respiratory syncytial virus infection. Viral Immunol. 2008;21(3):327-339.

45. Carson WE, et al. Interleukin (IL) 15 is a novel cytokine that activates human natural killer cells via components of the IL-2 receptor. J Exp Med. 1994;180(4):1395-1403.

46. Felices M, et al. Continuous treatment with IL-15 exhausts human NK cells via a metabolic defect. JCI Insight. 2018;3(3):96219.

47. Gong JH, Maki G, Klingemann HG. Characterization of a human cell line (NK-92) with phenotypical and functional characteristics of activated natural killer cells. Leukemia. 1994;8(4):652-658.

48. Mariotti FR, et al. PD-1 in human NK cells: evidence of cytoplasmic mRNA and protein expression. Oncoimmunology. 2019;8(3):1557030.

49. Denman CJ, et al. Membrane-bound IL-21 promotes sustained ex vivo proliferation of human natural killer cells. PLoS One. 2012;7(1):e30264.

50. Ciurea SO, et al. Phase 1 clinical trial using mbIL21 ex vivo-expanded donor-derived NK cells after haploidentical transplantation. Blood. 2017;130(16):1857-1868.

51. Canter RJ, et al. Radiotherapy enhances natural killer cell cytotoxicity and localization in pre-clinical canine sarcomas and first-in-dog clinical trial. J Immunother Cancer. 2017;5(1):98.

52. Park JS, et al. Canine cancer immunotherapy studies: linking mouse and human. J Immunother Cancer. 2016;4:97.

53. Liu Y, et al. Increased expression of programmed cell death protein 1 on NK cells inhibits NK-cell-mediated anti-tumor function and indicates poor prognosis in digestive cancers. Oncogene. 2017;36(44):6143-6153.

54. MacFarlane AW, et al. PD-1 expression on peripheral blood cells increases with stage in renal cell carcinoma patients and is rapidly reduced after surgical tumor resection. Cancer Immunol Res. 2014;2(4):320-331. 
55. Cho D, Shook DR, Shimasaki N, Chang YH, Fujisaki H, Campana D. Cytotoxicity of activated natural killer cells against pediatric solid tumors. Clin Cancer Res. 2010;16(15):3901-3909.

56. Tong AA, Hashem H, Eid S, Allen F, Kingsley D, Huang AY. Adoptive natural killer cell therapy is effective in reducing pulmonary metastasis of Ewing sarcoma. Oncoimmunology. 2017;6(4):e1303586.

57. Tallerico R, et al. Human NK cells selective targeting of colon cancer-initiating cells: a role for natural cytotoxicity receptors and MHC class I molecules. J Immunol. 2013;190(5):2381-2390.

58 . Mandal R, et al. The head and neck cancer immune landscape and its immunotherapeutic implications. JCI Insight. 2016;1(17):e89829.

59. Habif G, Crinier A, André P, Vivier E, Narni-Mancinelli $\mathrm{E}$. Targeting natural killer cells in solid tumors. Cell Mol Immunol. 2019;16(5):415-422.

60. Benson DM, et al. The PD-1/PD-L1 axis modulates the natural killer cell versus multiple myeloma effect: a therapeutic target for CT-011, a novel monoclonal anti-PD-1 antibody. Blood. 2010;116(13):2286-2294.

61. Abuzakouk M, Feighery C, O'Farrelly C. Collagenase and Dispase enzymes disrupt lympho- cyte surface molecules. JImmunol Methods. 1996;194(2):211-216.

62. Mulder WM, Koenen H, van de Muysenberg AJ, Bloemena E, Wagstaff J, Scheper RJ. Reduced expression of distinct T-cell CD molecules by collagenase/DNase treatment. Cancer Immunol Immunother. 1994;38(4):253-258.

63. Gallois A, Silva I, Osman I, Bhardwaj N. Reversal of natural killer cell exhaustion by TIM-3 blockade. Oncoimmunology. 2014;3(12):e946365.

64. Zhou J, et al. Liver-resident NK cells control antiviral activity of hepatic T cells via the PD-1PD-L1 axis. Immunity. 2019;50 (2):403-417.e4.

65. Raulet DH, Vance RE, McMahon CW. Regulation of the natural killer cell receptor repertoire. Annu Rev Immunol. 2001;19:291-330.

66. Narni-Mancinelli E, Vivier E, Kerdiles YM. The 'T-cell-ness' of NK cells: unexpected similarities between NK cells and T cells. Int Immunol. 2011;23(7):427-431.

67. Kerdiles Y, Ugolini S, Vivier E. T cell regulation of natural killer cells. J Exp Med. 2013;210(6):1065-1068.

68. Maecker HT, et al. Standardization of cytokine flow cytometry assays. BMC Immunol. 2005;6:13.

69. Metzger P, et al. Dying cells expose a nuclear anti- gen cross-reacting with anti-PD-1 monoclonal antibodies. Sci Rep. 2018;8(1):8810.

70. Caumartin J, et al. Trogocytosis-based generation of suppressive NK cells. EMBO J. 2007;26(5):1423-1433.

71. Miner CA, Giri TK, Meyer CE, Shabsovich M, Tripathy SK. Acquisition of activation receptor ligand by trogocytosis renders NK cells hyporesponsive. J Immunol. 2015;194(4):1945-1953.

72. Whiteside TL, Parmiani G. Tumor-infiltrating lymphocytes: their phenotype, functions and clinical use. Cancer Immunol Immunother. 1994;39(1):15-21.

73. Zhang $\mathrm{Q}$, et al. Blockade of the checkpoint receptor TIGIT prevents NK cell exhaustion and elicits potent anti-tumor immunity. Nat Immunol. 2018;19(7):723-732.

74. Somanchi SS, Senyukov VV, Denman CJ, Lee DA. Expansion, purification, and functional assessment of human peripheral blood NK cells. J Vis Exp. 2011;48(48):2540.

75. McCullen MV, Li H, Cam M, Sen SK, McVicar DW, Anderson SK. Analysis of Ly49 gene transcripts in mature NK cells supports a role for the Pro1 element in gene activation, not gene expression. Genes Immun. 2016;17(6):349-357. 